

\section{NOTICE}

This report was prepared as an account of work sponsored by an agency of the United States Government. Neither the United States Government nor any agency thereof, nor any of their employees, makes any warranty, express or implied, or assumes any legal liability or responsibility for the accuracy, completeness, or usefulness of any information, apparatus, product, or process disclosed, or represents that its use would not infringe privately owned rights. Reference herein to any specific commercial produce, process, or service by trade name, trademark, manufacturer, or otherwise, does not necessarily constitute or imply its endorsement, recommendation, or favoring by the United States Government or any agency thereof. The views and opinions of authors expressed herein do not necessarily state or reflect those of the United States Government or any agency thereof.

\section{NOTICE}

This report has been reproduced from the best available copy. Available in paper copy and microfiche.

Number of pages in this report: 45

DOE and DOE contractors can obtain copies of this report from:

Office of Scientific and Technical Information

P.O. Box 62

Oak Ridge, TN 37831 ;

(615) $576-8401$.

This report is publicly available from the:

National Technical Information Service

Department of Commerce

5285 Port Royal Road

Springfield, Virginia 22161

(703) $487-4650$ 


\section{DISCLAIMER}

Portions of this document may be illegible in electronic image products. Images are produced from the best available original document. 
October 15, 1994

\title{
Field-aligned Coordinates for Nonlinear Simulations of Tokamak Turbulence
}

\author{
M. A. Beer, S. C. Cowley, ${ }^{a)}$ and G. W. Hammett \\ Princeton University Plasma Physics Laboratory, P.O. Box 451, Princeton, NJ 08543
}

\begin{abstract}
Turbulence in tokamaks is characterized by long parallel wavelengths and short perpendicular wavelengths. We describe a coordinate system for nonlinear fluid, gyrokinetic "Vlasov", or particle simulations that exploits the elongated nature of the turbulence by resolving the minimum necessary simulation volume: a long thin twisting flux tube. It is very similar to the ballooning representation, although periodicity constraints can be incorporated in a manner that allows $\mathbf{E} \times \mathbf{B}$ nonlinearities to be evaluated efficiently with FFTs. If the parallel correlation length is very long, however, enforcing periodicity can introduce artificial correlations, so periodicity should not necessarily be enforced in poloidal angle at $\theta= \pm \pi$. We discuss the advantages and limitations of this approach, with application to 3D simulations of toroidal Ion Temperature Gradient (ITG) driven turbulence.
\end{abstract}

a) Present address: Dept. of Physics, UCLA, Los Angeles, CA 90024

PACS numbers: 52.35.Ra, 52.35.Qz, 52.55.Fa, 52.25.Fi

This article has been submitted to the journal Physics of Fluids.

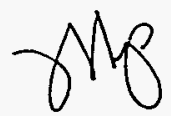

DIGTRIBUTION OF THIS DOCUMENT IS UNLIMITED

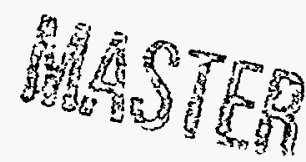




\section{Introduction}

The turbulence that evolves from fine-scale instabilities (e.g. $\eta_{i}$, trapped electron, or resistive ballooning modes) is thought to be responsible for the anomalously large particle, momentum, and heat transport levels in tokamaks. It is therefore of great interest to simulate numerically the nonlinear evolution of these instabilities to determine the resulting fluctuation and transport levels. These instabilities are characterized by long wavelengths parallel to the magnetic field and short perpendicular wavelengths, on the order of the ion gyroradius, $\rho_{i}$. This is, of course, a consequence of the rapid communication along field lines (at the sound speed for electrostatic instabilities) and slow communication across the field lines (typically velocities across the field do not exceed the diamagnetic speed). In addition, fluctuation measurements ${ }^{1,2}$ in tokamaks indicate a relatively short perpendicular correlation length $\left(\sim 10 \rho_{i}\right)$, but a long parallel correlation length. ${ }^{3}$ Simulation of a full tokamak with adequate resolution of these fine perpendicular scales is somewhat beyond the presently available computational resources, since $\rho_{i} / a \sim 10^{-3}$ for present day large tokamaks, where $a$ is the minor radius. (The latest full torus gyrokinetic particle simulations can now be run down to $\rho_{i} / a=1 / 128 .^{4}$ ) However, it may be unnecessary to simulate a whole torus to reproduce small-scale, locally-driven turbulence. This paper describes a coordinate system for nonlinear simulations that resolves a much smaller volume and is therefore computationally more efficient, while still resolving the relevant small scales. The smallest possible simulation volume is a long thin flux tube that is several correlation lengths wide in both perpendicular directions (radial and poloidal), and extended along the field line, exploiting the elongated nature of the turbulence $\left(k_{\perp} \gg k_{\|}\right)$. This approach is advantageous for fluid, gyrokinetic "Vlasov", and particle simulations, and could eventually be compared with full torus simulations.

The fundamental idea is to use coordinates that follow field lines. With such coordinates a flux tube (a tube with a surface parallel to $\mathbf{B}$ ) which is bent by magnetic curvature and twisted by magnetic shear, is mapped into a rectangular domain. Such twisting coordinates were originally proposed by Roberts and Taylor, ${ }^{5}$ and Cowley et al. ${ }^{6}$ emphasized their utility for nonlinear calculations. In Ref. 7, we described the essential features of this approach, with an emphasis on slab geometry. Here we focus more on the toroidal aspects and actual details of implementation. 
The major problem of these field line coordinates is enforcing the periodicity constraint since the coordinates are multivalued in a torus (except at low order rational surfaces). In Ref. 6 it was emphasized that it is unlikely that the correlated volume wraps around the torus and overlaps itself. When this is true, the physical periodicity of the full torus is irrelevant, and the simplest approach is to simulate a flux tube subdomain that is several parallel correlation lengths long (just as it should be several perpendicular correlation lengths wide). As will be described in Sec. III, this can be different from imposing periodicity at $\theta= \pm \pi$ as is usually suggested for the ballooning representation (and which could lead to artificial correlations which modify the results).

Another advantage of the field-line coordinates, in addition to the efficiency of a minimum simulation volume, is that it can easily implement radial periodicity (which in regular coordinates is complicated by the shear of the magnetic field), thus avoiding the problems of "quasilinear flattening" and allowing self-consistent turbulence-generated "zonal" flows (flows which cause flux surfaces to rotate). The field-line coordinates are also particularly convenient for gyrofluid simulations where partially Fourier transformed quantities (in 2 of the 3 dimensions) need to be evaluated, such as $\left|\omega_{d}(\theta)\right| \propto\left|k_{\theta} \cos (\theta)+k_{\tau} \sin (\theta)\right|$.

We have carried out simulations with various sizes for the flux-tube "box", and verified that the results are independent of the box size (once it is larger that the correlation lengths in each direction), thus justifying some of the assumptions implicit in simulating a flux tube subdomain rather than the full torus. This leads to interesting questions regarding Bohm vs. gyro-Bohm scaling for the turbulence, which we will will consider in the conclusions.

In section II we describe the general formulation of the basic geometry. The issues of periodicity and parallel boundary conditions are discussed in detail in section III. Parallel boundary conditions for particle simulations are presented in section IV. Section V discusses the relation of these flux tube coordinates to the standard ballooning transformation. In section VI we specialize to an axisymmetric low- $\beta$ tokamak equilibrium. We present some simulation results for ITG turbulence using this coordinate system in section VII, and investigate the effect of the parallel boundary conditions. In section VIII we discuss these results, the computational efficiency of flux tube simulation, and possible limitations of this approach. For completeness the equations used in the 
simulations are included in the Appendix.

\section{Flux tube simulations in general}

If one wants to describe turbulence which is highly elongated along field lines and narrowly localized across field lines it is natural to introduce coordinates which are constant on field lines. A natural way to do this for any general magnetic field is to use the Clebsch representation of the magnetic field ${ }^{8}($ since $\nabla \cdot \mathbf{B}=0)$ :

$$
\mathbf{B}=\nabla \alpha \times \nabla \psi
$$

Clearly $\mathbf{B} \cdot \nabla \alpha=\mathbf{B} \cdot \nabla \psi=0$ so that $\alpha$ and $\psi$ are constant on field lines. Thus $\alpha$ and $\psi$ are natural coordinates for the flux tube. A third coordinate, $z$, must be defined that represents distance along the flux tube. One obvious choice of the third coordinate is the physical length along the field line, though this is not always the most convenient choice. A complication of using $\alpha$ and $\psi$ as coordinates is that they are not unique, for instance if $\alpha^{\prime}=\alpha+g(\psi)$ then $\mathbf{B}=\nabla \alpha^{\prime} \times \nabla \psi$. In many applications toroidal flux surfaces are defined and it is natural to take $\psi$ to be the poloidal flux. The choice of $\alpha$ is less obvious and may be optimized for a particular calculation. A further complication is that $\alpha$ and $\psi$ are typically not naturally single valued and a cut must be introduced to enforce single values. ${ }^{8}$ This issue will be discussed extensively below. Let us imagine that a choice of $\alpha, \psi$, and $z$ has been made and that $\alpha=\alpha(\mathbf{r}), \psi=\psi(\mathbf{r})$, and $z=z(\mathbf{r})$ are known functions. This information can be obtained for instance from the output of an equilibrium code. Thus the metric coefficients for the transformation to the $\alpha, \psi, z$ coordinates are taken to be known. The Jacobian of this transformation is $J=(\nabla \alpha \times \nabla \psi \cdot \nabla z)^{-1}$.

Three spatial operators appear many times in the equations for the perturbations, they are: $\mathbf{B} \cdot \nabla, \nabla_{\perp}^{2}$, and $\mathbf{B} \times \nabla \Phi \cdot \nabla$. In the $\alpha, \psi, z$ coordinates we have:

$$
\begin{gathered}
\text { B } \cdot \nabla A=(\nabla \alpha \times \nabla \psi \cdot \nabla z)\left(\frac{\partial A}{\partial z}\right)_{\alpha, \psi}=\frac{1}{J} \frac{\partial A}{\partial z} \\
\nabla^{2} A=\frac{1}{J} \frac{\partial}{\partial \alpha}\left[J \frac{\partial A}{\partial \alpha}|\nabla \alpha|^{2}+J \frac{\partial A}{\partial \psi} \nabla \alpha \cdot \nabla \psi+J \frac{\partial A}{\partial z} \nabla \alpha \cdot \nabla z\right] \\
+\frac{1}{J} \frac{\partial}{\partial \psi}\left[J \frac{\partial A}{\partial \alpha} \nabla \alpha \cdot \nabla \psi+J \frac{\partial A}{\partial \psi}|\nabla \psi|^{2}+J \frac{\partial A}{\partial z} \nabla \psi \cdot \nabla z\right]
\end{gathered}
$$




$$
\begin{aligned}
&+\frac{1}{J} \frac{\partial}{\partial z}\left[J \frac{\partial A}{\partial \alpha} \nabla \alpha \cdot \nabla z+J \frac{\partial A}{\partial \psi} \nabla \psi \cdot \nabla z+J \frac{\partial A}{\partial z}|\nabla z|^{2}\right] \\
& \mathbf{B} \times \nabla \Phi \cdot \nabla A=\left(\frac{\partial A}{\partial \psi} \frac{\partial \Phi}{\partial \alpha}-\frac{\partial A}{\partial \alpha} \frac{\partial \Phi}{\partial \psi}\right) B^{2} \\
&+\left(\frac{\partial A}{\partial z} \frac{\partial \Phi}{\partial \alpha}-\frac{\partial A}{\partial \alpha} \frac{\partial \Phi}{\partial z}\right)(\nabla \alpha \times \nabla \psi) \cdot(\nabla \alpha \times \nabla z) \\
&+\left(\frac{\partial A}{\partial z} \frac{\partial \Phi}{\partial \psi}-\frac{\partial A}{\partial \psi} \frac{\partial \Phi}{\partial z}\right)(\nabla \alpha \times \nabla \psi) \cdot(\nabla \psi \times \nabla z)
\end{aligned}
$$

where $A$ and $\Phi$ are any scalars. Eqs. (2)-(4) are completely general.

We shall assume that the turbulence we wish to simulate has perpendicular correlation lengths that are short compared to equilibrium scale lengths but a parallel correlation length of the same order as the equilibrium scale lengths. Let us consider a simulation domain that is a flux tube volume defined by $\alpha_{0}-\Delta \alpha<\alpha<\alpha_{0}+\Delta \alpha, \psi_{0}-\Delta \psi<\psi<\psi_{0}+\Delta \psi$, and $-z_{0}<z<z_{0}$. This volume is chosen to be several correlation lengths in all three directions. Of course one wants to make this volume as small as possible to save computer time. Once the box volume is larger than several correlation lengths the turbulence should be insensitive to the size of the box. One tests whether the box size is adequate (in the usual way) by increasing the box size and comparing the turbulence in the different size boxes, or by measuring the correlation functions in a given box and verifying that they go to zero at the edges of the box. In this way we arrive at a minimum simulation volume.

Since the simulation volume is narrow in $\alpha$ and $\psi$ (compared to equilibrium variations) all equilibrium quantities (or gradients of equilibrium quantities when they appear in the equations) are to lowest order functions of $z$ alone. In other words, the perpendicular scale of the equilibrium is much greater than the perpendicular scale of the perturbations, and the box is chosen to be only slightly larger than the largest scale perturbations, so across the box (i.e. in $\alpha$ and $\psi$ ) one can ignore the variation of these equilibrium quantities. For example, the Jacobian $J=(\nabla \alpha \times \nabla \psi \cdot \nabla z)^{-1}$ is to a good approximation constant across the box but not along the box, thus $J=J\left(\alpha_{0}, \psi_{0}, z\right)$.

When $A$ is a perturbed scalar (e.g. $n, T$, etc.), and $\Phi$ is the potential, we can neglect the $\partial / \partial z$ terms in Eqs. (3) and (4) since they are smaller by $k_{\|} / k_{\perp}$. The coefficients in Eqs. (2), (3), and (4) (various elements of the metric tensor) are again roughly constant across the box and therefore 
may be taken as functions of $z$ alone with $\alpha=\alpha_{0}$ and $\psi=\psi_{0}$. Then Eqs. (3) and (4) reduce to:

$$
\begin{gathered}
\nabla_{\perp}^{2} A=|\nabla \alpha|^{2} \frac{\partial^{2} A}{\partial \alpha^{2}}+2 \nabla \alpha \cdot \nabla \psi \frac{\partial^{2} A}{\partial \alpha \partial \psi}+|\nabla \psi|^{2} \frac{\partial^{2} A}{\partial \psi^{2}} \\
\mathbf{B} \times \nabla \Phi \cdot \nabla A=\left(\frac{\partial A}{\partial \psi} \frac{\partial \Phi}{\partial \alpha}-\frac{\partial A}{\partial \alpha} \frac{\partial \Phi}{\partial \psi}\right) B^{2} .
\end{gathered}
$$

Therefore, the equations to be solved in this (minimum simulation) volume have no explicit dependence on $\alpha$ or $\psi$, which leads to great computational simplification. The $\mathbf{E} \times \mathbf{B}$ nonlinearity takes the simple form Eq. (6), and all other coefficients in the equations are only functions of $z$.

The perpendicular boundary conditions on the perturbations at $\alpha=\alpha_{0} \pm \Delta \alpha$ and $\psi=\psi_{0} \pm$ $\Delta \psi$ are taken to be periodic. If the box is more than a correlation length wide the turbulence should be insensitive to the boundary conditions, although one set of boundary conditions that is not advisable is fixed boundary conditions which prohibit energy and particle fluxes through the boundary. If fixed radial boundary conditions without sources or sinks are used, then the $m=0, n=0$ component of the perturbations (where $m$ is the poloidal mode number and $n$ is the toroidal mode number) will grow to eventually cancel the driving equilibrium gradients ("quasilinear flattening"), thus turning off the turbulence. In principle, this problem can be overcome with a sufficiently large box so that the time scale to flatten the driving gradients becomes much longer than the simulation time. But periodic radial boundary conditions avoid flattening altogether and allow the use of a more efficient, smaller box. Past simulations have sometimes zeroed out the $m=0, n=0$ components of perturbations to avoid this flattening, but that prevents the turbulence from being able to generate sheared zonal flows (resulting from the $m=0, n=0$ component of the electrostatic potential, $\Phi(\psi)$, which varies only with minor radius), which can be an important nonlinear saturation process. ${ }^{7,9-13}$ Periodic radial boundary conditions allow the self-consistent evolution of $m=0, n=0$ perturbations such as the zonal flows.

The assumption of radial periodicity in the small flux-tube is not based on actual physical constraints (that would require simulating the full tokamak to include losses to the limiter, auxiliary heating of the tokamak core, and including a vacuum region and a conducting shell). Instead, we are assuming that the statistical properties of the fluctuations at $\psi+2 \Delta \psi$ are the same as at $\psi$, and that if the simulation box width $2 \Delta \psi$ is larger than the radial correlation length we can assume that 
they are actually identical at every instant. This statistical radial periodicity also serves as a model of the effect of turbulence in neighboring regions on the simulated subdomain. This is illustrated by the contours in Fig. 9, which show eddies that stick out of one side of the box and reenter on the other side of the box. Periodic boundary conditions are often used in 2-D plasma simulations (such as Hasegawa-Mima) or in simulations of homogeneous Navier-Stokes Turbulence, but it is complicated somewhat in 3-D plasma simulations by the shear in the magnetic field. Because the parallel dynamics are so much faster than the perpendicular dynamics (so $k_{\|} \ll k_{\perp}$ ), the fluctuations tend to be elongated along the direction of the magnetic field, which points in different directions at different radii. In regular coordinates this requires the use of something like the "twist-andshift" radial boundary conditions suggested by Kotschenreuther and Wong. ${ }^{9,14,15}$ The field-line coordinates, however, are already aligned with the magnetic field, so radial periodicity becomes simply $A(\psi+2 \Delta \psi, \alpha, z, t)=A(\psi, \alpha, z, t)$. Some of the issues involved in radial periodicity are discussed in more detail in Refs. $(9,16,10)$.

For the same reasons, we can also assume statistical periodicity in the $\alpha$ direction, $A(\psi, \alpha+$ $2 \Delta \alpha, z, t)=A(\psi, \alpha, z, t)$. There is no explicit dependence of the operators in Eqs. $(5,6)$ on $\alpha$ or $\psi$, so it is useful to expand in a Fourier series in $\psi$ and $\alpha$ (which also provides periodicity in those directions):

$$
A(\psi, \alpha, z, t)=\sum_{j=-\infty}^{\infty} \sum_{k=-\infty}^{\infty} \hat{A}_{j, k}(z, t) e^{i j \pi\left(\psi-\psi_{0}\right) / \Delta \psi+i k \pi\left(\alpha-\alpha_{0}\right) / \Delta \alpha} .
$$

The boundary conditions in the $z$ direction will be discussed in the next section. Note that while each term in the Fourier series is a plane wave in $\alpha, \psi$ coordinates, the wavefronts in real space can be very distorted. Perhaps the most pronounced distortion arises from magnetic shear. To understand this we first define the angle, $\lambda$, between constant $\alpha$ and $\psi$ surfaces:

$$
\cos \lambda=\frac{\nabla \alpha \cdot \nabla \psi}{|\nabla \alpha||\nabla \psi|}
$$

Magnetic shear makes $\lambda$ change as $z$ changes -in real space the flux tube is then sheared and its cross-section goes from being rectangular where $\lambda=\pi / 2$ to being a parallelogram where $\lambda \neq \pi / 2$, as shown in Fig. 1. The wavefronts of each term in the Fourier series, Eq. (7), also get sheared. For example the $j=0, k \neq 0$ term has wavefronts corresponding to the constant $\alpha$ lines. The 
individual terms in the series Eq. (7) are therefore "twisted eddies" 5,6 whose wavefronts twist as one moves along $z$.
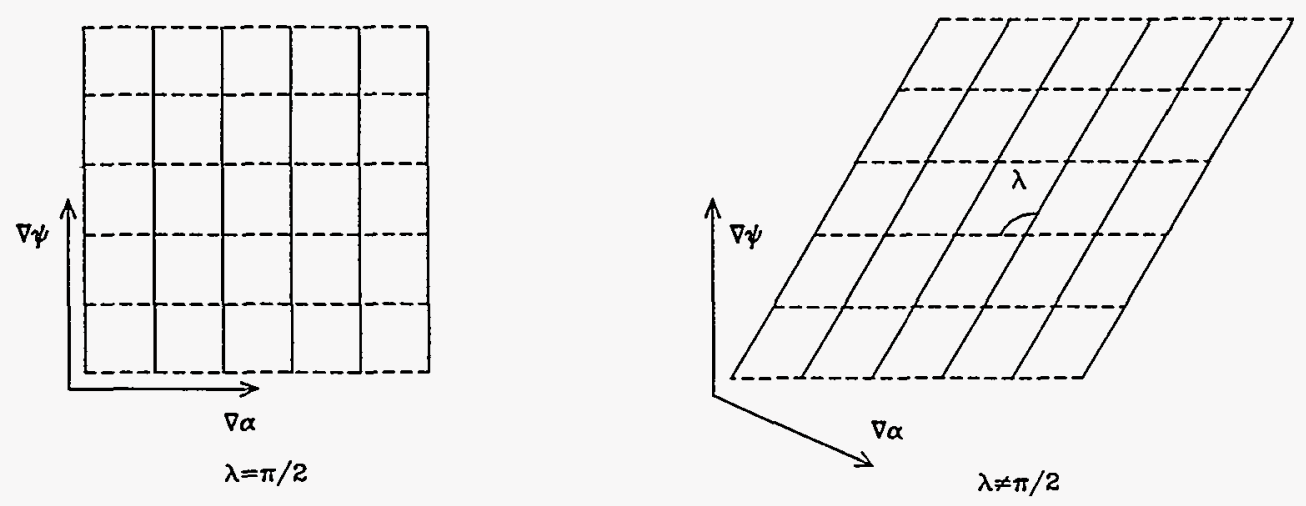

Figure 1: Shearing of flux tube cross section at different positions along the tube. Lines represent constant $\psi$ (dashed) and constant $\alpha$ (solid) lines.

Now let us discuss the choice of the coordinates $\alpha$ and $\psi$. A useful discussion of this procedure can be found in Ref. 17. As shown in Ref. 18, it is possible to choose $\alpha, \psi$, and generalized "toroidal" and "poloidal" angle variables $\zeta$ and $\theta$ such that the field lines are straight in the $(\zeta, \theta)$ plane and physical quantities are periodic over $2 \pi$ in both variables. This choice of coordinates will simplify our discussion of periodicity in section III. For the general magnetic field Eq. (1), we have: ${ }^{8}$

$$
\alpha=\phi-q(\psi) \theta-\nu(\psi, \theta, \phi)
$$

where $\psi=(2 \pi)^{-2} \int_{V} d \tau \mathbf{B} \cdot \nabla \theta$ is the poloidal flux, $q(\psi)=d \psi_{T} / d \psi, \psi_{T}=(2 \pi)^{-2} \int_{V} d \tau \mathbf{B} \cdot \nabla \phi$ is the toroidal flux, $d \tau$ is the volume element, and $\phi$ and $\theta$ are the physical toroidal and poloidal angles, so physical quantities are periodic over $2 \pi$ in $\phi$ and $\theta$. The function $\nu$ is also periodic in $\phi$ and $\theta$. We now introduce a new toroidal coordinate,

$$
\zeta=\phi-\nu(\psi, \theta, \phi)
$$


With this choice

$$
\alpha=\zeta-q(\psi) \theta
$$

and the magnetic field lines are straight in the $(\zeta, \theta)$ plane. Further, periodicity is preserved in $\zeta$ and $\theta$. Often, $\theta$ is also redefined to choose a specific form of the Jacobian. An alternative to Eq. (10) would be to use $\zeta=\phi$ and introduce a new poloidal coordinate $\theta^{\prime}=\theta+\nu / q$. In any case, we will make use of the fact that a coordinate system can be chosen such that magnetic fields lines are straight in the $(\theta, \zeta)$ plane, and are given by $\alpha=\zeta-q(\psi) \theta=$ constant. For our parallel coordinate $z$ we will use $z=\theta$, since this makes our description very close to the usual ballooning mode formalism. Note that $z$ is not restricted to $-\pi<z<\pi$, as we may choose to simulate a flux tube which follows a field line wrapping around the torus several times in the poloidal direction, not just once. This will be discussed further in the next section.

In summary, our field-line following coordinate system is given by $(\psi, \alpha, z)$, where field lines are labeled by constant $\psi$ and $\alpha$. One can think of $\psi$ as a radial coordinate, $\alpha=\zeta-q(\psi) \theta$ as a perpendicular-to-the-field coordinate, and $z=\theta$ as a parallel-to-the-field coordinate. Our notation simplifies if we introduce the following new variables:

$$
x=\frac{q_{0}}{B_{0} r_{0}}\left(\psi-\psi_{0}\right), \quad y=-\frac{r_{0}}{q_{0}}\left(\alpha-\alpha_{0}\right), \quad z=\theta,
$$

where $q_{0}=q\left(\psi_{0}\right), B_{0}$ is the field at the magnetic axis, and $r_{0}$ is the distance from the magnetic axis to the center of the box. Then Eq. (7) becomes:

$$
A(x, y, z, t)=\sum_{k_{x}=-\infty}^{\infty} \sum_{k_{y}=-\infty}^{\infty} e^{i k_{x} x+i k_{y} y} \hat{A}_{k_{x}, k_{y}}(z, t),
$$

with $k_{x}=j \pi / \Delta x, k_{y}=-k \pi / \Delta y, \Delta x=q_{0} \Delta \psi / B_{0} r_{0}$, and $\Delta y=r_{0} \Delta \alpha / q_{0}$. The rectangular computational box of "radial" width $2 \Delta x$, and "poloidal" width $2 \Delta y$, and extended along the field line, $\theta$, is mapped onto a flux tube, as shown in Fig. 2, for example.

These coordinates are similar to those used in Ref. 19. Our $\alpha, \psi$, and $z$ are analogous to $-q \theta^{\prime}$, $\rho^{\prime}$, and $\phi^{\prime}$ in Ref. 19, respectively, since they have chosen to measure the distance along the field line with $\phi^{\prime}$, a "toroidal" angle, while we use $\theta$. A more significant difference between our representation and Ref. 19 is the treatment of periodicity, though their more recent work ${ }^{20}$ has adopted a similar treatment to ours, described in the next section. 


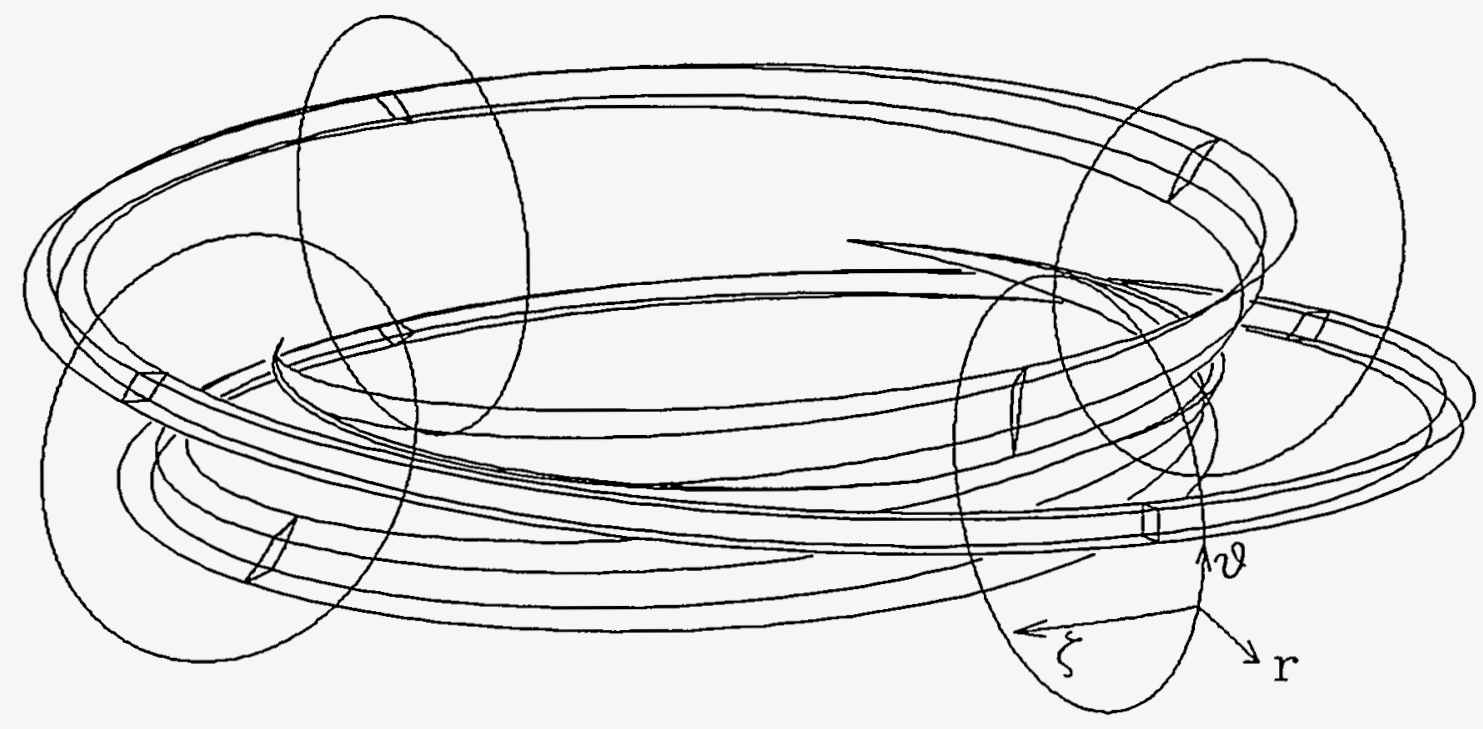

Figure 2: The rectangular computational domain mapped onto a flux tube in a torus, with $q_{0}=2.4$ and shear, $\hat{s}=1.5$. The ends of this flux tube are cut off at poloidal angle $-\pi$ and $\pi$, and the sheared cross-sections of the flux tube in the poloidal plane are indicated. 


\section{Periodicity and parallel boundary conditions}

The choice of parallel boundary conditions involves a number of subtle, yet important issues. The main concept is that of a statistically-motivated periodicity, as described in Sec. II for the $\psi$ and $\alpha$ boundary conditions. For moderately "ballooning" turbulence we might expect parallel correlation lengths $\theta_{c} \sim(1-2) \pi$ (though it might be longer than this). The simulation box should have a length $2 z_{0}=2 \pi N$ in the parallel direction which is several times the parallel correlation length. In some cases a box length of $2 \pi$ might be sufficient. But an even longer box may be necessary in many cases to ensure that one end of the box is sufficiently decorrelated from the other end of the box to avoid artificially constraining correlation effects, just as the box must be at least a few correlation lengths wide in the $\psi$ and $\alpha$ directions. For the cases simulated in Sec. VII, parallel box lengths of at least $4 \pi$ were needed for good convergence.

One must be careful about which other coordinates are held fixed while applying parallel periodicity, just as one must be careful to impose radial periodicity in field-line coordinates $(\psi, \alpha, z)$ (i.e., impose periodicity in $\psi$ while holding $\alpha$ and $z$ fixed). As discussed in Sec. II, trying to impose radial periodicity in the usual $(\psi, \theta, \zeta)$ coordinates would miss the fact that fluctuations tend to be extended along the magnetic field, which changes direction in the the $(\theta, \zeta)$ plane as $\psi$ is varied. Similarly, though the flux-tube is rectangular in $(\psi, \alpha)$ coordinates, it twists into a parallelogram in physical space as one follows the flux-tube along $z$ (Figs. 1 and 2). The fluctuations in the physical plane perpendicular to a magnetic field line should be statistically identical at all places along that field-line with the same poloidal angle $(z=0,2 \pi, 4 \pi, \ldots)$, irrespective of the twisting of the flux-tube which increases without bound as $z \rightarrow \infty$. Because of this, we will assume that the fluctuations are periodic in $z$ while holding $(\psi, \zeta)$ fixed, rather than holding the field-line coordinates $(\psi, \alpha)$ fixed. The reader may find it easier to visualize this in sheared slab geometry, as carried out in Ref. 7.

A related problem is that if we were to impose parallel periodicity as $A\left(\psi, \alpha,+z_{0}\right)=A\left(\psi, \alpha,-z_{0}\right)$, then every field line would effectively be a rational field line that connected to itself. Field lines are labeled by constant $(\psi, \alpha)$, and such a boundary condition causes any particles flowing out one end of the field line to flow back in the other end of the box on the same field line. This is unlike a real 
sheared magnetic field where the set of irrational field-lines is dense, i.e., most of the field-lines are irrational and never connect to themselves.

So, we will impose periodicity in $z$ while holding $\psi$ and $\zeta$ fixed (rather than holding $\psi$ and $\alpha$ fixed). Specifically,

$$
\left.A(\psi, \alpha(\theta, \zeta), z(\theta))\right|_{\theta=N \pi}=\left.A(\psi, \alpha(\theta, \zeta), z(\theta))\right|_{\theta=-N \pi}
$$

Rather than the form of a boundary condition in $z$, this can be stated as a more general periodicity relation with period $2 \pi N$ :

$$
A(\psi, \alpha(\theta+2 \pi N, \zeta), z(\theta+2 \pi N))=A(\psi, \alpha(\theta, \zeta), z(\theta))
$$

Physically, this is equivalent to considering two $(\psi, \zeta)$ planes cutting through the flux tube, at $z=\theta$ and at $z=\theta+2 \pi N$, and assuming that the turbulence is (statistically) identical in those two planes. To evaluate this periodicity constraint, first substitute $\alpha=\zeta-q(\psi) \theta, z=\theta$ into Eq. (7), and take $\alpha_{0}=0$ for simplicity (it drops out), to get

$$
A=\sum_{j=-\infty}^{\infty} \sum_{k=-\infty}^{\infty} \hat{A}_{j, k}(\theta, t) e^{i j \pi\left(\psi-\psi_{0}\right) / \Delta \psi+i k \pi \zeta / \Delta \alpha-i k \pi q(\psi) \theta / \Delta \alpha} .
$$

For a thin flux-tube, we can approximate $q(\psi) \approx q_{0}+\left(\psi-\psi_{0}\right) q^{\prime}$, where $q^{\prime} \equiv(\partial q / \partial \psi)_{\psi=\psi_{0}}$, to get

$$
A=\sum_{j=-\infty}^{\infty} \sum_{k=-\infty}^{\infty} \hat{A}_{j, k}(\theta, t) e^{i \pi\left(\psi-\psi_{0}\right)\left(j / \Delta \psi-k q^{\prime} \theta / \Delta \alpha\right)+i k \pi \zeta / \Delta \alpha-i k \pi q_{0} \theta / \Delta \alpha} .
$$

Substituting this into Eq. (14) yields

$$
\begin{gathered}
\sum_{j=-\infty}^{\infty} \sum_{k=-\infty}^{\infty} \hat{A}_{j, k}(\theta+2 \pi N, t) e^{i \pi\left(\psi-\psi_{0}\right)\left(j / \Delta \psi-k q^{\prime}(\theta+2 \pi N) / \Delta \alpha\right)+i k \pi \zeta / \Delta \alpha-i k \pi q_{0}(\theta+2 \pi N) / \Delta \alpha} \\
=\sum_{j=-\infty}^{\infty} \sum_{k=-\infty}^{\infty} \hat{A}_{j, k}(\theta, t) e^{i \pi\left(\psi-\psi_{0}\right)\left(j / \Delta \psi-k q^{\prime} \theta / \Delta \alpha\right)+i k \pi \zeta / \Delta \alpha-i k \pi q_{0} \theta / \Delta \alpha} .
\end{gathered}
$$

In order for this to be valid at any arbitrary value of $\zeta$, the coefficient of each $\exp (i k \pi \zeta / \Delta \alpha)$ term must be identical:

$$
\begin{gathered}
\sum_{j=-\infty}^{\infty} \hat{A}_{j, k}(\theta+2 \pi N, t) e^{i \pi\left(\psi-\psi_{0}\right)\left(j / \Delta \psi-k q^{\prime}(\theta+2 \pi N) / \Delta \alpha\right)-i k \pi q_{0}(\theta+2 \pi N) / \Delta \alpha} \\
=\sum_{j=-\infty}^{\infty} \hat{A}_{j, k}(\theta, t) e^{i \pi\left(\psi-\psi_{0}\right)\left(j / \Delta \psi-k q^{\prime} \theta / \Delta \alpha\right)-i k \pi q_{0} \theta / \Delta \alpha}
\end{gathered}
$$


We can make the coefficients of $\left(\psi-\psi_{0}\right)$ identical by shifting the $j$ index with the substitution $j=j^{\prime}+\delta j$ into the left-hand side, where

$$
\delta j=2 \pi N k q^{\prime} \Delta \psi / \Delta \alpha=2 \pi N k \Delta q / \Delta \alpha
$$

and where $2 \Delta q=2 q^{\prime} \Delta \psi$ is the change in $q$ from one edge of the box to the other. Note that $\delta j$ must be an integer, which quantizes the ratio $\Delta q / \Delta \alpha$, as discussed below. We now have

$$
\begin{gathered}
\sum_{j^{\prime}=-\infty}^{\infty} \hat{A}_{j^{\prime}+\delta j, k}(\theta+2 \pi N, t) e^{i \pi\left(\psi-\psi_{0}\right)\left(j^{\prime} / \Delta \psi-k q^{\prime} \theta / \Delta \alpha\right)-i k \pi q_{0}(\theta+2 \pi N) / \Delta \alpha} \\
=\sum_{j=-\infty}^{\infty} \hat{A}_{j, k}(\theta, t) e^{i \pi\left(\psi-\psi_{0}\right)\left(j / \Delta \psi-k q^{\prime} \theta / \Delta \alpha\right)-i k \pi q_{0} \theta / \Delta \alpha}
\end{gathered}
$$

For convenience, we can take the width of the simulation volume $2 \Delta \alpha$ to be $1 / n_{0}$ of the circumference in the toroidal direction,

$$
\Delta \alpha=\pi / n_{0}
$$

where $n_{0}$ is a positive integer. Dropping the primes on $j$ in Eq. (20), the parallel periodicity condition now becomes

$$
\begin{array}{ll}
\hat{A}_{j+\delta j, k}(\theta+2 \pi N, t) C_{k} & =\hat{A}_{j, k}(\theta, t), \\
\delta j=k J, & J=2 n_{0} N \Delta q,
\end{array}
$$

where the phase-factor $C_{k}=\exp \left(-i 2 \pi N k q_{0} n_{0}\right)$. Note that the requirement that $j$ be an integer quantizes the range of $q$ spanned by the flux tube $2 \Delta q$ to be $J / n_{0} N$, where $J$ is an integer. For $q^{\prime} \neq 0$, this then quantizes the radial box size since $\Delta q=q^{\prime} \Delta \psi$. One can treat shearless $q^{\prime}=0$ cases as well, then $\delta j=J=0$, and the radial box size $2 \Delta \psi$ is no longer quantized and just needs to be at least a few radial correlation lengths wide. In the usual $q^{\prime} \neq 0$ case, the radial position of the simulation box can always be adjusted slightly (less than one radial box width) so that $q_{0}=q\left(\psi_{0}\right)$ is rational such that the phase-factor $C_{k}=1$.

Eq. (22) thus expresses a modified periodicity condition on the mode amplitudes: the value of a coefficient at one end of the box is specified by the value of another coefficient (with the same $k$ but a different $j$, i.e., a different $\theta_{0}$, as we will describe below) at the other end of a box. This is represented graphically in Fig. 3 (which uses notation introduced below). Of course computer 
simulations can not retain an infinite set of $j$ 's and $k$ 's. Instead, enough $j$ and $k$ modes are kept to be able to resolve up to a desired value of $k_{\perp} \rho_{i}$, above which the coefficients $\hat{A}_{j, k}$ are assumed to vanish. Note that $\delta j=0$ for $k=0$ modes, so the periodicity condition for $k=0$ modes simplifies to $\hat{A}_{j, 0}(\theta+2 \pi N, t)=\hat{A}_{j, 0}(\theta, t)$.

This completes the formal specification of the boundary conditions, but we go on to express it in terms of notation often used in the ballooning transformation. It is common to introduce the "ballooning angle" $\theta_{0}(j, k)$, such that the radial derivative of an individual $(j, k)$ mode of Eq. (16),

$$
\left.\frac{\partial}{\partial \psi}\right|_{\theta, \zeta} \propto i \pi\left(j / \Delta \psi-k q^{\prime} \theta / \Delta \alpha\right)
$$

vanishes at $\theta=\theta_{0}$. Note that this definition of $\theta_{0}$ employs a derivative with respect to $\psi$ while holding $\theta$ and $\zeta$ fixed, not $\alpha$ and $\theta$. Clearly at $\theta=\theta_{0}(j, k)$ the wavefronts of the $j, k^{\prime}$ th term in Eq. (7) are perpendicular to the $\psi$ surfaces. Eqs. (21-24) yield

$$
k \theta_{0}(j, k)=\frac{j \Delta \alpha}{\Delta \psi q^{\prime}}=\frac{j \pi}{n_{0} \Delta q} .
$$

$\theta_{0}$ is discrete with spacing $\delta \theta_{0}=j \pi / k n_{0} \Delta q$ that is dependent on $k$. Only the combination $k \theta_{0}$ ever appears and the limit $k=0$ must be interpreted in terms of the discrete $j$ sum. In particular, the turbulence can generate $k=0\left(\theta_{0}=\infty\right)$ modes corresponding to zonal flows which can be important in the nonlinear dynamics, so the $k=0$ modes must be allowed to evolve self-consistently. (Likewise, one must be careful about the shearless limit $q^{\prime}=0$, where $\theta_{0} \rightarrow \infty$. The field-line coordinates are still useful, but it is then better to think about the $j$ (or $k_{x}$ ) label of the mode, which remains finite, rather than the $\theta_{0}$ label.) Using the definition of $\theta_{0}$ in Eq. (25), we can express the shift $\delta j$ in Eq. (19) as a shift in $\theta_{0}$ instead:

$$
\Delta \theta_{0}=\frac{\delta j \pi}{k n_{0} \Delta q}=2 \pi N
$$

Using the definition of $\theta_{0}$ to denote $\hat{A}_{j, k}$ by a corresponding $\hat{A}_{\theta_{0}, k}$, and absorbing a phase factor which is independent of the coordinates $(\psi, \theta, \zeta)$ by using $\bar{A}_{j, k}=\hat{A}_{j, k} \exp \left[-i k n_{0}\left(q_{0} \theta_{0}(j, k)+\alpha_{0}\right)\right]$, the parallel periodicity condition of Eq.(22) can be written in a form related to the familiar ballooning representation,

$$
\bar{A}_{\theta_{0}+2 \pi N, k}(\theta, t)=\bar{A}_{\theta_{0}, k}(\theta-2 \pi N, t) .
$$


This form of the periodicity relation is illustrated graphically in Fig. 3.

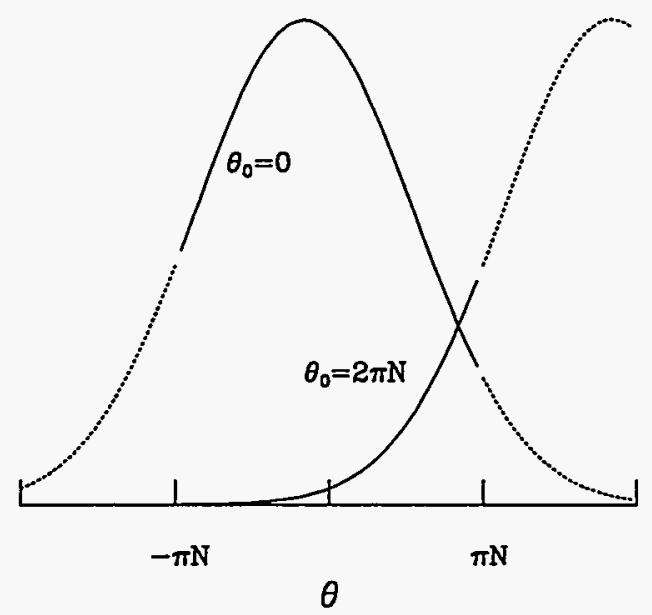

Figure 3: Boundary condition for $\bar{A}$ along the field line coordinate, Eq. (27). Different $k_{x}$ modes (i.e. $\theta_{0}$ 's) with the same $k_{y}$ are connected at $\pm \pi N$, i.e. $\bar{A}_{\theta_{0}, k}(-\pi N)$ is connected to $\bar{A}_{\theta_{0}+2 \pi N, k}(\pi N)$.

Using Eqs. (21) and (25) and $q(\psi) \approx q_{0}+\left(\psi-\psi_{0}\right) q^{\prime}$ (or going back to Eq. (7) and using $q$ itself for the radial-like coordinate $\psi$ ), we can rewrite Eq. (16) as

$$
A(\psi, \theta, \zeta, t)=\sum_{j=-\infty}^{\infty} \sum_{k=-\infty}^{\infty} \bar{A}_{j, k}(\theta, t) e^{i k n_{0}\left[\zeta-q(\psi)\left(\theta-\theta_{0}(j, k)\right)\right]}
$$

It should be emphasized that Eqs. (16) and (28) are merely the same equations in different notation. Eq. (28) bears a strong resemblance to the standard ballooning representation. There are however important differences that we will discuss more fully in section $\mathrm{V}$.

Eq. (28), when used with the periodicity relation in Eq. (27), is periodic in $\theta$ with period $2 \pi N$. By setting $N=1$, this can satisfy physical periodicity in $\theta$, achieving the same result as the "sum over p" in the standard ballooning representation (see Eq. (32)). Thus, we are able to recover physical periodicity as does the quasiballooning approach. ${ }^{15}$ However, one should not necessarily use $N=1$. Rather, one should use a large enough $N$ so that the parallel box length $2 z_{0}=2 \pi N$ is at least several times the parallel correlation length, as argued in the beginning of this section. This point may be confusing to those who think that $N=1$ regular periodicity in $\theta$ should always 
be enforced because $\theta$ is a physical variable. This would be true if we were simulating the full torus with $n_{0}=1$. Indeed, Eq. (28) or (16) provides an expansion in a complete basis set if $n_{0}=1$ and $N=1$. However, we are not trying to simulate the full torus, but a thin flux-tube whose width is only $1 / n_{0}$ of the full toroidal circumference (motivated by the short wavelengths and short perpendicular correlation lengths of the turbulence, as discussed in the introduction). Then Eq. (28) represents $n_{0}$ identical copies of the simulation volume if one considers the full range of $\zeta, 0 \rightarrow 2 \pi$. The distance along the field line in this simulation volume is parameterized by $z=\theta$. Following the flux tube along the field lines (at fixed $\alpha=\zeta-q(\psi) \theta$ ) from $\theta=0$ to $\theta=2 \pi$ will not lead to the same physical location (unless $q$ is very close to an integer) but to one of the $n_{0}-1$ identical copies of itself. Forcing periodicity at this point is undesirable (unless the parallel correlation length is indeed significantly shorter than $2 \pi$ ) because it is a fiction of simulating only $1 / n_{0}$ of the toroidal direction with $n_{0}$ identical copies.

This is illustrated by Fig. 4, which shows a correlated volume with a parallel correlation length $\theta_{c} \approx 3 \pi$, and a perpendicular correlation length equal to half the simulation box width, $\alpha_{c}=$ $\Delta \alpha=\pi / 6$. If the simulation flux tube has a parallel length of only $2 \pi$, then this correlated volume would be forced to overlap with one of the $n_{0}$ images of itself, causing artificial interference effects. By extending the simulated flux tube to a length of $4 \pi$, we allow the whole region to evolve self-consistently.

Of course, at an integer $q$ flux surface, a simulation volume really does overlap itself within a distance $\theta=2 \pi$ and experience these interference effects. More generally, a correlated volume will overlap itself when $\theta$ increases by $2 \pi N$ if $q 2 \pi N$ modulo $2 \pi$ is less than the perpendicular correlation length $\alpha_{c}$. This can be used to define a maximum parallel length $\theta_{\max }$ which the flux-tube can be without physically overlapping itself. $\theta_{\max }$ is also the maximum correlation length a correlated perturbation can have without "biting it's tail" and experiencing coherent interference effects. $\theta_{\max }$ is plotted vs. $q(\psi)$ in Fig. 5 . Note that if one simulates only $1 / n_{0}$ of the toroidal direction, then a correlated perturbation is $n_{0}$ times as likely to run into itself or one of its images. In this case we may need to extend the parallel length of the simulated flux-tube to avoid these artificial correlations. For most of the plasma, there is no difficulty in extending the simulated flux-tube to 


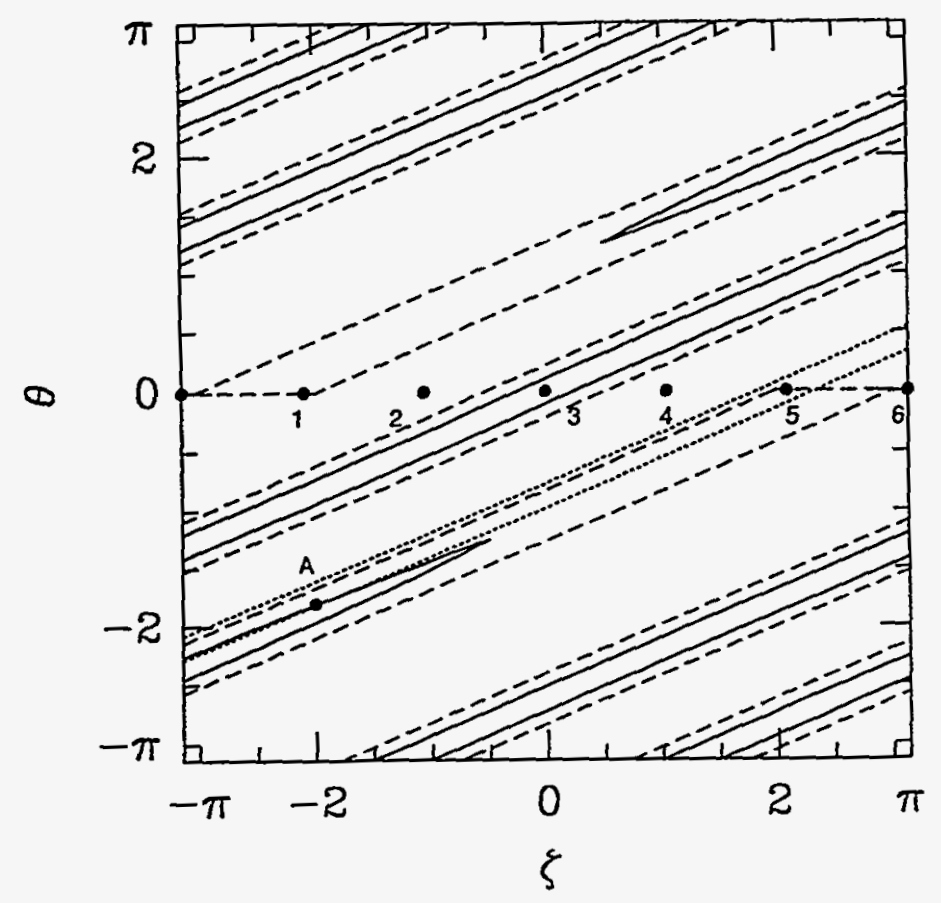

Figure 4: Tlustration on a flux surface of a possible correlated volume of the point 3 (enclosed by the solid line, with parallel correlation length $\theta_{c} \approx 3 \pi$ ), and a minimum simulation volume enclosed by the dashed line. The diagonal lines are parallel to the field lines (here $q=2.4$ ). In this case the simulation volume has a toroidal width of one sixth the total toroidal circumference, i.e. $n_{0}$ in Eq. (21) is 6 . If the potential is represented by Eq. (28) and $\Phi$ is made periodic in $\theta$, there are six identical copies of the correlated volume centered at the points 1-6. The correlated volume of point 5 (dotted line) partially overlaps the correlated volume of point 3 , at the point marked $A$. This is unphysical and can be avoided in this case by making the system periodic over $4 \pi,-2 \pi<\theta<2 \pi$. The minimum simulation volume illustrated is for $-2 \pi<\theta<2 \pi$. 
be 2-3 times longer than $2 \pi$, without having the flux-tube physically run in to itself. Even for a simulation flux tube which spans a range of $q$ values, for example $2 \Delta q \sim 1 / 2$, at worst the flux tube might overlap itself briefly near an integer or half-integer $q$ surface. As pointed out in Ref. (6), these low-order rational surfaces occupy a small fraction of a minor radius of a tokamak and so it is very infrequent that a correlated perturbation will "bite it's tail". Furthermore, experimental evidence ${ }^{21}$ on tokamaks indicates that there are no unusual features near low-order rational surfaces (except when there are macroscopic MHD instabilities).

a)

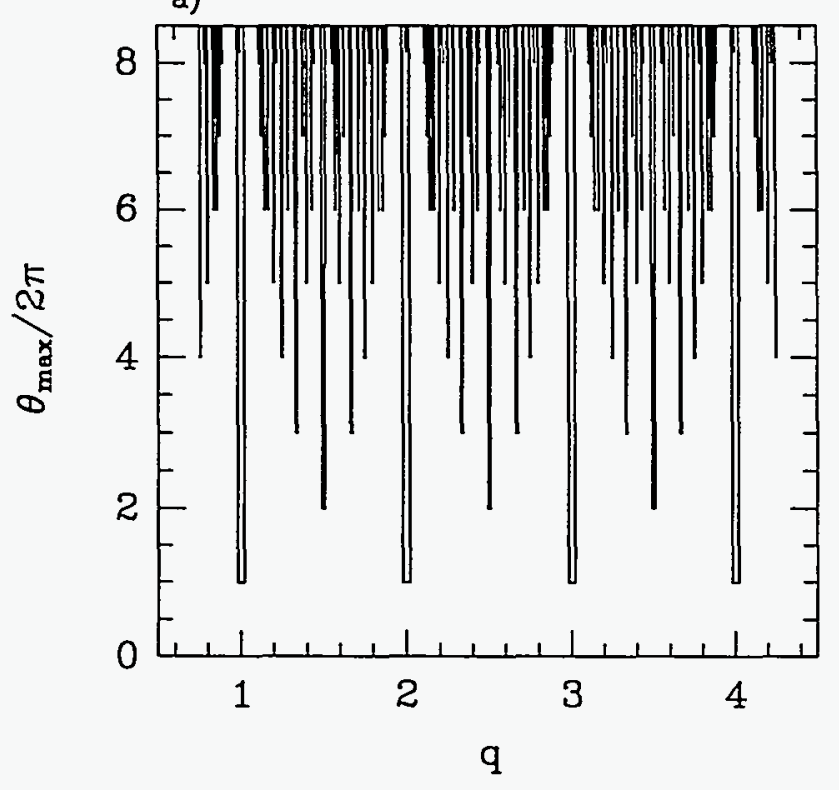

b)

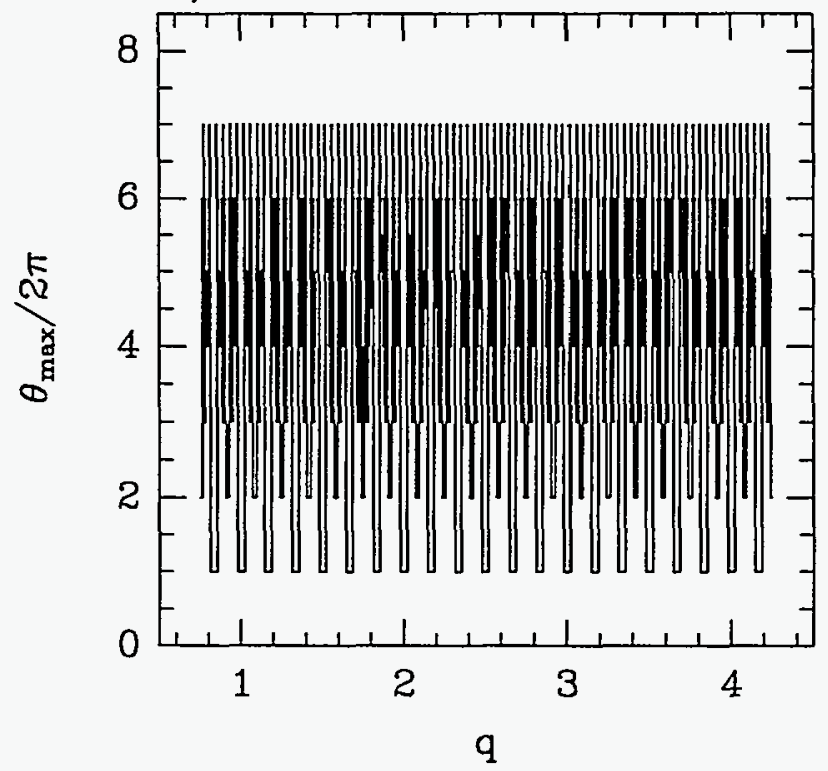

Figure 5: Distance along the field line, $\theta_{\max }$, at which a correlated volume (with perpendicular width $2 \Delta \alpha=\pi / 25$ ) overlaps itself, for varying $q$. a) For $n_{0}=1, \theta_{\max }$ is small only near low order $q$ surfaces. b) For $n_{0}=6$, the maximum correlation length is reduced, since the correlated volume can hit copies of itself. In this case, if the physical correlation length is longer than $\theta_{\text {max }}$, the box must be extended and the periodicity condition relaxed.

In practice we find that the flux-tube length $2 \pi N$ doesn't need to be extremely large, and $N=2$ may usually be sufficient. For the particular cases used in Sec. VII, (Figs. 12 and 13), we find that $N=1$ simulations produce a $\chi_{i}$ which is about $30 \%$ low, while $N=2-4$ are virtually indistinguishable. However, there may be other cases where an even larger $N$ is required. In 
each case, one should justify the value of $N$ a posteriori, by verifying that the parallel correlation functions from the simulations indeed fall off significantly in a distance $2 \pi N$, and/or by carrying out convergence studies with different values of $N$ (just as convergence with the size of the box in the other 2 directions should also be studied). Again, the fundamental assumption in all of this is that it is probably sufficient (and most efficient) to use a simulation volume which is just a few correlation lengths in all 3 directions.

\section{Boundary conditions for particle simulations}

Particle simulations can also take advantage of an optimum flux-tube simulation volume using the field-line coordinates $(\psi, \alpha, z)$ described in Sec. III. Field quantities such as the electrostatic potential can be represented by the Fourier series Eq. (7), with the parallel boundary conditions given by Eq. (22), or equivalently, Eq. (27).

For the particles, we must specify the location where a particle will reenter the box after passing through an edge of the box. The particle's velocity should not be changed. In the perpendicular directions $\psi$ and $\alpha$, standard periodicity is used. In the parallel direction, $z$, periodicity is applied while holding $\psi$ and $\zeta$ fixed (rather than holding the field-line coordinates $\psi$ and $\alpha$ fixed), for the reasons described at the beginning of Sec. III. To quantify this, first recall the definitions $\alpha=\zeta-q(\psi) \theta$, and $z=\theta$. If a particle exits the box at the position $\left(\psi_{1}, \alpha_{1}, z=+\pi N\right)$, where $\alpha_{1}=\zeta_{1}-q\left(\psi_{1}\right) \pi N$, then it will reenter the opposite side of the box at $\left(\psi_{2}, \alpha_{2}, z=-\pi N\right)$, where $\psi_{2}=\psi_{1}$, and $\alpha_{2}=\zeta_{1}+q\left(\psi_{1}\right) \pi N$. Thus the particle will be shifted in $\alpha$ by the amount

$$
\delta \alpha=\alpha_{2}-\alpha_{1}=q\left(\psi_{1}\right) 2 \pi N \text { modulo } 2 \Delta \alpha
$$

Where the modulo operation accounts for the fact that if this shift in $\alpha$ causes $\alpha_{2}$ to fall outside the range of the box, $-\Delta \alpha<\alpha<\Delta \alpha$, then the particle has fallen into a periodic copy of the original box, and is simply shifted by a multiple of $2 \Delta \alpha$ back into the simulation domain. Expanding $q(\psi)=q_{0}+\left(\psi-\psi_{0}\right) q^{\prime}$, using Eqs.(21) and (23), and introducing an integer $K$ to reproduce the $2 K \Delta \alpha$ shift of the modulo function, we find that

$$
\frac{\delta \alpha(\psi)}{2 \Delta \alpha}=q_{0} N n_{0}+K+J \frac{\left(\psi-\psi_{0}\right)}{2 \Delta \psi}
$$


As discussed after Eq. (23), $q_{0} N n_{0}$ can usually be assumed to be an integer. At the outer edge of the box, $\psi=\psi_{0}+\Delta \psi$, the box has twisted by $J / 2$ box lengths in the $\alpha$ direction, and by $-J / 2$ box lengths at the inner edge of the box, $\psi=\psi_{0}-\Delta \psi$. Thus $J$ represents the integer number of box widths in $\alpha$ that the box has twisted from one end in $z$ to the other end. This is illustrated for $J=2$ in Fig. 6 . In this figure, $q_{0} N n_{0}$ is assumed to be an integer for simplicity, so the center of the box is at the same physical point at $\theta= \pm \pi N$. In general, the ends of the box will overlap with periodic copies of the original box. (It may be easier to visualize this in a box which spans $\psi_{0}<\psi<\psi_{0}+2 \Delta \psi$, rather then being centered around $\psi_{0}$. Then the inner edge of the box at $\psi_{0}$ is stationary, and the outer edge at $\psi_{0}+2 \Delta \psi$ will be twisted by $J$ box widths.)

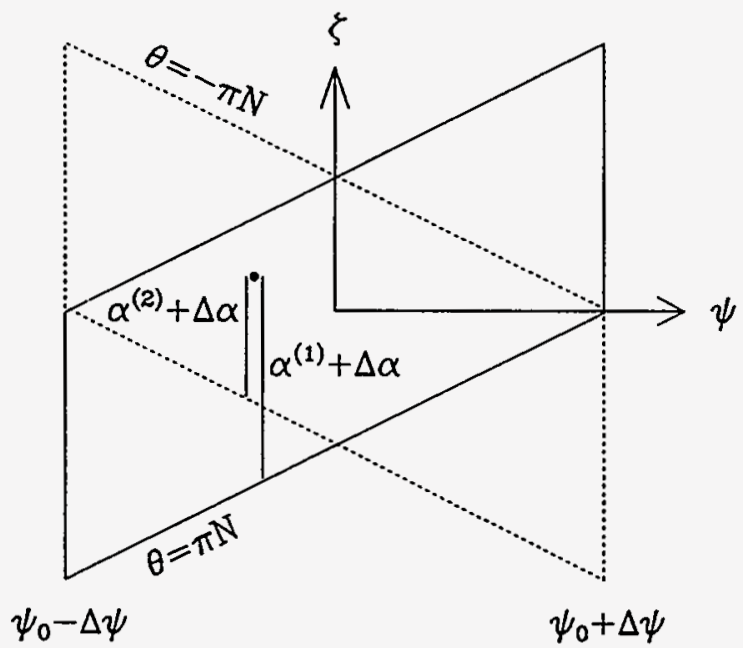

Figure 6: Boundary conditions in the parallel direction. At $\theta=0$, the simulation box is rectangular in $\zeta$ and $\psi$. The twisted ends of the box at $\theta=\pi N$ (solid) and $\theta=-\pi N$ (dashed) are shown. If a particle leaves the $\theta=\pi N$ end of the box at $\alpha^{(1)}$, it reenters the $\theta=-\pi N$ end of the box at $\alpha^{(2)}$, given by Eq. (29).

To summarize, if a particle:

\begin{tabular}{|c|c|}
\hline leaves the box from & then it reenters at \\
\hline$\left(\psi_{0}+\Delta \psi, \alpha, z\right)$ & $\left(\psi_{0}-\Delta \psi, \alpha, z\right)$ \\
$(\psi,+\Delta \alpha, z)$ & $(\psi,-\Delta \alpha, z)$ \\
$(\psi, \alpha,+\pi N)$ & $(\psi, \alpha+\delta \alpha,-\pi N)$ \\
\hline
\end{tabular}


The equivalent particle boundary conditions can also be stated for the $(x, y, z)$ coordinates of Eq. (12). If a particle leaves the end of the box at $\left(x_{1}, y_{1}, z=+\pi N\right)$, it reenters at $\left(x_{1}, y_{2}, z=\right.$ $-\pi N)$, where

$$
\delta y=y_{2}-y_{1}=-\left(q_{0} N n_{0} 2 \Delta y+J \Delta y x / \Delta x\right) \text { modulo } 2 \Delta y
$$

the analogue of Eq. (29). The integer $J=2 \pi N \hat{s} \Delta x / \Delta y$, (with $\left.\hat{s} \equiv\left(r_{0} / q_{0}\right)(\partial q / \partial r)_{r=r_{0}}\right)$ measures the number of twists of the box in the $y$ direction from one end in $z$ to the other. Thus, if a particle:

\begin{tabular}{|c|c|}
\hline leaves the box from & then it reenters at \\
\hline$(\Delta x, y, z)$ & $(-\Delta x, y, z)$ \\
$(x, \Delta y, z)$ & $(x, \Delta y, z)$ \\
$(x, y,+\pi N)$ & $(x, y+\delta y,-\pi N)$ \\
\hline
\end{tabular}

Of course all of the above boundary conditions are reversible, i.e., if a particle leaves at $(-\Delta x, y, z)$, it will reenter at $(\Delta x, y, z)$, etc.

\section{The Ballooning Transformation and its relation to flux tube simulation}

The linear theory of short perpendicular wavelength instabilities in tokamaks has been developed largely in terms of the so called "Ballooning Transformation." 22 In this section we will discuss the relationship of the "Ballooning Transformation" to our flux tube simulation scheme. In Ballooning theory a single eigenmode is represented as:

$$
\Phi_{n}(\psi, \theta, \zeta, t)=\sum_{p=-\infty}^{\infty} e^{-i \omega t+i n \zeta-i n q(\psi)\left(\theta-\theta_{0}+2 \pi p\right)} \hat{\Phi}_{n, \theta_{0}}(\theta+2 \pi p, \psi),
$$

where $\theta_{0}=\theta_{0}(\psi)$ and $\hat{\Phi}_{n, \theta_{0}}(\theta, \psi)$ depend on $\psi$. The toroidal mode number $n$ is any large integer. The variation in $\theta$ and $\psi$ of the exponential is large whereas the variation of $\theta_{0}$ and $\hat{\Phi}$ is finite. In lowest order in an expansion in $1 / n q$ one obtains a differential equation in $\theta$ for $\hat{\Phi}_{n, \theta_{0}}(\theta, \psi)$. This equation is solved with $\theta_{0}$ a parameter and with the boundary conditions $\hat{\Phi} \rightarrow 0$ as $|\theta| \rightarrow \infty$, so the sum over $p$ can converge. Periodicity in $\theta$ is recovered by the $p$ summation in Eq. (32). A lowest order approximation to the eigenvalue $\omega_{n}\left(\theta_{0}, \psi\right)$ is obtained on each surface. In higher order the eigenvalue is quantized by solving radial differential equations. Much has been written about this higher order procedure to find the radial behavior and we cannot do justice to the subtleties here. ${ }^{23}$ Let us consider instead a narrow radial annulus $\psi_{0}-\Delta \psi<\psi<\psi_{0}+\Delta \psi$. Let $\Phi$ be periodic in 
$\psi$ over $2 \Delta \psi$ at constant $\alpha=\zeta-q(\psi) \theta$ and $\theta$; then we can represent the radial variation of $\Phi$ in a Fourier series in $\psi$, with $n \theta_{0}=l \pi / \Delta q$, i.e. the $\psi$ variation of $\theta_{0}(\psi)$ and $\hat{\Phi}_{n, \theta_{0}}(\theta, \psi)$ are combined into a discrete series in $\theta_{0}$. Thus one could write for an arbitrary perturbation in this annulus:

$$
\Phi(\psi, \theta, \zeta, t)=\sum_{n=-\infty}^{\infty} \sum_{l=-l_{0}+1}^{l_{0}} \sum_{p=-\infty}^{\infty} e^{i n \zeta-i n q(\psi)(\theta+2 \pi p)+i l \pi\left(\psi-\psi_{0}\right) / \Delta \psi} \bar{\Phi}_{n, l}(\theta+2 \pi p, t),
$$

where we have rescaled $e^{i l q_{0} \pi / q^{\prime} \Delta \psi} \hat{\Phi}_{n, l}=\bar{\Phi}_{n, l}$. The $p$ summation makes this expression manifestly periodic in $\theta$. Expanding $q(\psi)$, so $\exp \left[-i n q 2 \pi p+i l \pi\left(\psi-\psi_{0}\right) / \Delta \psi\right]=\exp \left[-i n q_{0} 2 \pi p+i \pi(l-\right.$ $\left.2 p n \Delta q)\left(\psi-\psi_{0}\right) / \Delta \psi\right]$, it is clear that in this summation we need only take $|l| \leq l_{0}=n \Delta q$ since otherwise the $p$ and $l$ sums duplicate terms. This restricts the bandwidth in $\psi$ of the perturbations in ballooning space, and makes the ballooning transformation unique. ${ }^{24}$

If we set $n_{0}=1$ in Eq. (28) and $N=1$ in Eqs. (22) and (23) we obtain an exactly equivalent representation to Eq. (33). To see this we note that the $j$ in Eq. (28) and $p$ and $l$ in Eq. (33) are related by $j=l-2 p l_{0}$ and $\delta j=2 l_{0}$, and we set $k=n$. Thus the $-\pi<\theta<\pi$ range of the $\bar{\Phi}_{n, l}$ modes with $|l|<l_{0}$ correspond to the $\bar{A}_{j, k}$ modes with $|j|<\delta j / 2$ (defined only from $-\pi<\theta<\pi$ for $N=1$ ). The $\bar{A}_{j, k}$ modes with $|j|>\delta j / 2$ correspond to the $-\pi-2 \pi p<\theta<\pi-2 \pi p$ range of the $\bar{\Phi}_{n, l}$ modes with $p=(j-l) / \delta j$. The boundary condition Eq. (22) makes this series of $\bar{A}_{j, k}$ modes (for all $j$ ) identical to $\bar{\Phi}_{n, l}$ (for $|l|<l_{0}$ ) defined on the extended domain $-\infty<\theta<\infty$ (when $n_{0}=N=1$ ).

The boundary condition Eq. (22) simplifies the evaluation of the $\mathbf{E} \times \mathbf{B}$ nonlinearities compared to the usual ballooning representation. The simple form Eq. (6) is easy to evaluate using a pseudospectral method. A fully spectral method remains in $\mathbf{k}$ space at all times, so the nonlinear terms become convolutions in $\mathrm{k}$ space and require of order $N_{x}^{2} N_{y}^{2} N_{z} \sim N^{5}$ operations. By using Fast Fourier Transforms (FFTs), the pseudospectral method reduces the operations to $N_{x} N_{y} N_{z}\left(\log _{2} N_{x}+\log _{2} N_{y}\right) \sim N^{3}$ resulting in a very significant savings for large $N$.

In the ballooning representation (i.e. using Eq. (33) to represent the perturbations) the nonlinear terms involve sums over $p:^{25}$

$$
\left(\mathrm{v}_{E} \cdot \nabla A\right)_{n, l}(\theta)=\frac{c}{2} \sum_{n^{\prime}+n^{\prime \prime}=n} \sum_{l^{\prime}} \sum_{p^{\prime}, p^{\prime \prime}} e^{-2 \pi i q_{0}\left(n^{\prime} p^{\prime}+n^{\prime \prime} p^{\prime \prime}\right)} n^{\prime} n^{\prime \prime} q^{\prime}\left[2 \pi\left(p^{\prime \prime}-p^{\prime}\right)+\theta_{0}^{\prime}-\theta_{0}^{\prime \prime}\right] \times
$$




$$
\left[\bar{\Phi}_{n^{\prime}, l^{\prime}}\left(\theta+2 \pi p^{\prime}\right) \bar{A}_{n^{\prime \prime}, l^{\prime \prime}}\left(\theta+2 \pi p^{\prime \prime}\right)-\bar{A}_{n^{\prime}, l^{\prime \prime}}\left(\theta+2 \pi p^{\prime}\right) \bar{\Phi}_{n^{\prime \prime}, l^{\prime \prime}}\left(\theta+2 \pi p^{\prime \prime}\right)\right]
$$

where $l^{\prime \prime}=l-l^{\prime}+2 \Delta q\left(n^{\prime} p^{\prime}+n^{\prime \prime} p^{\prime \prime}\right)$ and $\theta_{0}(n, l)=l \pi / n \Delta q$. Again $\left|l^{\prime}\right| \leq\left|n^{\prime}\right| \Delta q$ and $\left|l^{\prime \prime}\right| \leq\left|n^{\prime \prime}\right| \Delta q$, and $\bar{A}$ and $\bar{\Phi}$ are defined on an infinitely extended $\theta$ domain, without the boundary condition Eq. (22). This expression differs slightly from earlier literature since we are using a discrete representation in $\psi$, and have implicitly used the inverse ballooning transformation. ${ }^{24}$ If the mode width in $\theta$ is less than $\pi$, the sums over $p$ appear to be a small effect, and are usually neglected in nonlinear calculations using the ballooning representation. This conclusion may be misleading. Noting that in Eq. (33) $k_{x}=j \pi / \Delta x=\left(l-2 p l_{0}\right) \pi / \Delta x$ and $k_{y}=-n \pi / \Delta y=-n q_{0} / r_{0}$, we see that in the standard ballooning representation, only a wedge of $\bar{\Phi}_{n, l}$ 's in $\left(k_{x}, k_{y}\right)$ space are evolved, $-n \Delta q<l<n \Delta q$ (for $n \neq 0$ ), and the rest of $\mathrm{k}$ space is filled by the sum over $p$. For small $n$ the range of $k_{x}$ 's evolved is small, so it may take many terms in the $p$ sum to reach moderate $k_{x}$ 's. The wedge of modes evolved in the ballooning representation are the open circles in Fig. 7, while our approach evolves a rectangle of modes in $k_{x}$ and $k_{y}$, up to $k_{y} \rho_{i} \approx 1$ and at least $k_{x} \rho_{i} \approx 1$ (both the circles and the dots in Fig. 7). This figure corresponds to the mode arrangement of the runs in Fig. 13, where the shear is very weak $(\hat{s}=0.1), \Delta x \approx 1.4 \Delta y, k_{y}^{\max } \rho_{i} \approx 1$, and $k_{x}^{\max } \rho_{i} \approx 1.2$, so $J=N$. The nonlinear interaction between a mode $\left(k_{x}, k_{y}\right)$ within the $p=0$ wedge and a mode outside the wedge (the square box in Fig. 7, for example) could be strong, even if its linearly most unstable mode structure (of many eigenmodes in $\theta$ ) is centered a long distance down the field line. For low $k_{y}$ and large $k_{x}$ one would have to include many $p$ 's to capture this interaction (in this case, nine). In our nonlinear simulations, we do see modes outside the $p=0$ wedge excited to significant amplitudes.

While the usual $\mathbf{k}_{\perp}{ }^{\prime} \times \mathbf{k}_{\perp}{ }^{\prime \prime} \cdot \hat{\mathbf{b}}$ nonlinearity can be efficiently evaluated pseudospectrally, it is not obvious that the ballooning nonlinearity, with its sums over $p$, can be. However, since our representation is equivalent to the ballooning representation (if $n_{0}=N=1$ ), it automatically includes the sums over $p$ in the nonlinearity. Thus the most efficient way to numerically evaluate the nonlinear terms using the ballooning representation, if one were forced to, is probably to break the $\theta$ domain into segments of $2 \pi$, fill a rectangle in $\left(k_{x}, k_{y}\right)$ space with the sum over $p$, and apply the pseudospectral method to Eq. (6). Our representation automatically accomplishes all of this. Our representation should also be more convenient for analytic calculations, since the nonlinearity 


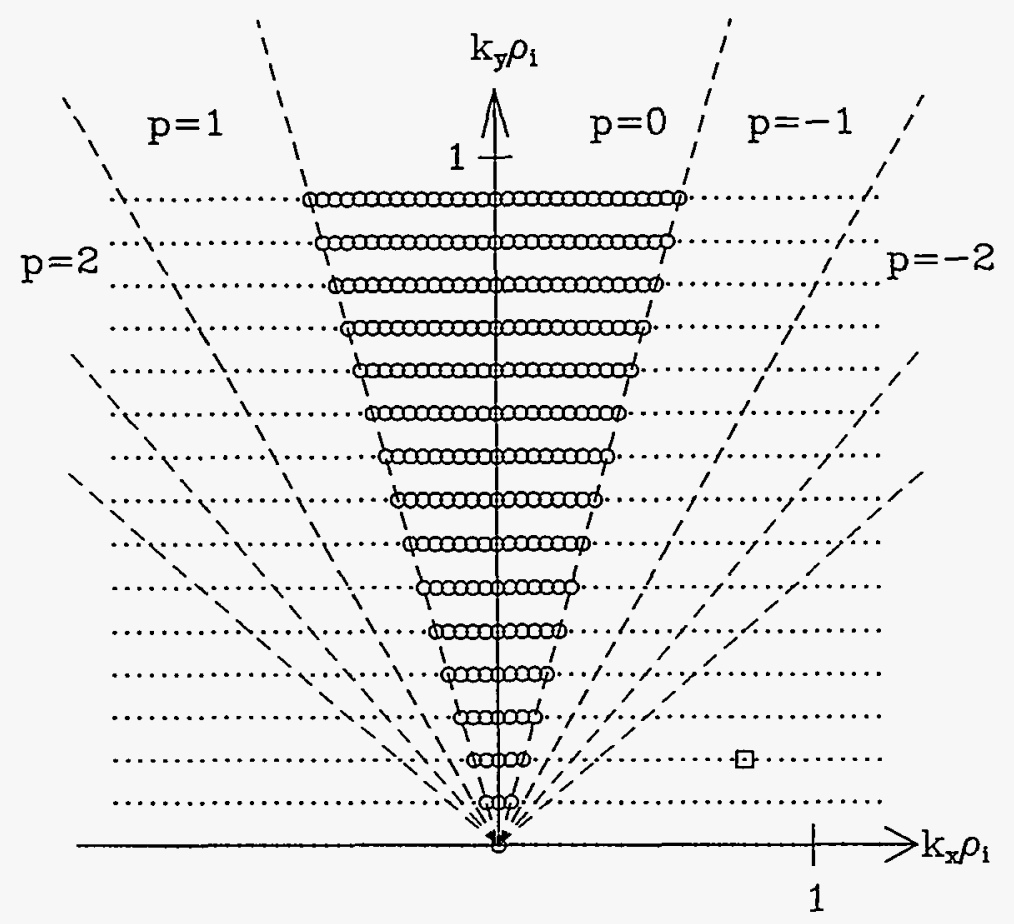

Figure 7: The wedge of $k_{x}, k_{y}$ modes evolved in the ballooning representation (open circles), and the rectangle of modes evolved in our approach (circles and dots). To recover the nonlinear interaction between the $p=0$ modes and the mode marked by the square box, nine terms in the $p$ sum of Eq. (34) are needed, even though $k_{x} \rho_{i} \approx 0.8$ for this mode.

takes a simple form, and the choice of $\theta_{0}$ 's, or $k_{x}$ 's, is well defined.

\section{Axisymmetric low- $\beta$ equilibrium}

We now specialize to the case of a low- $\beta$, large aspect ratio, axisymmetric torus with circular concentric flux surfaces. In the usual $r, \theta, \phi$, coordinates (minor radius, poloidal angle, and toroidal angle), $\nu=-\left(q r / R_{0}\right) \sin \theta$ in Eq. (10), and

$$
\mathbf{B}=B\left(\hat{\mathbf{e}}_{\phi}+\frac{r}{q R_{0}} \hat{\mathbf{e}}_{\theta}\right)
$$

where $B=B_{0} R_{0} / R, R$ is the distance from the axis of symmetry, and $R_{0}$ is the major radius. The $\zeta$ defined in Eq. (10) is the usual toroidal angle, $\phi$, to lowest order in $r / R_{0}$; we will only keep terms to lowest order in $r / R_{0}$ here. Near $\psi_{0}$, we can expand $\psi-\psi_{0}=\int_{r_{0}}^{r} d r B_{0} r / q \simeq\left(r-r_{0}\right) B_{0} r_{0} / q_{0}$. 
Then Eq. (12) becomes, choosing $\alpha_{0}=0$ :

$$
x=r-r_{0}, \quad y=\frac{r_{0}}{q_{0}}[q(r) \theta-\phi], \quad z=\theta .
$$

In these variables, the parallel derivative becomes, using Eq. (2) with $J^{-1} \simeq q_{0} R_{0} / B_{0}$,

$$
\hat{\mathrm{b}} \cdot \nabla A=\frac{1}{q_{0} R_{0}} \frac{\partial A}{\partial \theta}
$$

and the perpendicular gradient is:

$$
\nabla_{\perp} A=\left(\frac{\partial A}{\partial x}+\hat{s} \theta \frac{\partial A}{\partial y}\right) \hat{\mathrm{e}}_{r}+\frac{\partial A}{\partial y} \hat{\mathrm{e}}_{\theta}
$$

The linear $\omega_{*}$ terms, arising from $\mathbf{E} \times \mathbf{B}$ convection of the equilibrium, using Eq. (6), are:

$$
\mathbf{v}_{E} \cdot \nabla A_{0}=\frac{c}{B^{2}} \mathbf{B} \times \nabla \Phi \cdot \nabla A_{0}=c \frac{\partial A_{0}}{\partial \psi} \frac{\partial \Phi}{\partial \alpha}=-\frac{c}{B_{0}} \frac{\partial A_{0}}{\partial r} \frac{\partial \Phi}{\partial y}
$$

The nonlinear $\mathbf{E} \times \mathbf{B}$ terms are:

$$
\mathbf{v}_{E} \cdot \nabla A=\frac{c}{B_{0}}\left(\frac{\partial \Phi}{\partial x} \frac{\partial A}{\partial y}-\frac{\partial \Phi}{\partial y} \frac{\partial A}{\partial x}\right)
$$

Using Eq. (5), with $|\nabla \alpha|^{2} \simeq q_{0}^{2}\left(1+\hat{s}^{2} \theta^{2}\right) / r_{0}^{2}, \nabla \alpha \cdot \nabla \psi \simeq-B_{0} \theta \hat{s}$, and $|\nabla \psi| \simeq B_{0} r_{0} / q_{0}$ :

$$
\nabla_{\perp}^{2} A=\frac{\partial^{2} A}{\partial y^{2}}\left(1+\hat{s}^{2} \theta^{2}\right)+2 \hat{s} \theta \frac{\partial^{2} A}{\partial x \partial y}+\frac{\partial^{2} A}{\partial x^{2}} .
$$

Using the definition of $\theta_{0}$ in Eq. (25), $k_{x}=-k_{y} \hat{s} \theta_{0}$,

$$
\nabla_{\perp}^{2} A=-k_{y}^{2} A\left[1+\hat{s}^{2}\left(\theta-\theta_{0}\right)^{2}\right]
$$

That this takes the usual ballooning form should come as no surprise, given the discussion in section V. The combined $\nabla B$ and curvature drifts can be written:

$$
\mathbf{v}_{d}=\frac{v_{\|}^{2}+v_{\perp}^{2} / 2}{\Omega B^{2}} \mathbf{B} \times \nabla B+\frac{4 \pi v_{\|}^{2}}{\Omega B^{2}} \hat{\mathbf{b}} \times \nabla p,
$$

where the $\nabla p$ term is negligible in this low- $\beta$ equilibrium. For an axisymmetric $\mathbf{B}, \partial B / \partial \alpha=$ 0 ; for our low- $\beta$ equilibrium we also have $\partial B / \partial \psi \simeq-\left(q_{0} / r_{0} R_{0}\right) \cos \theta, \partial B / \partial z \simeq\left(B_{0} r_{0} / R_{0}\right) \sin \theta$, $\mathbf{B} \cdot \nabla \alpha \times \nabla z \simeq-\left(B_{0} q_{0} / r_{0}^{2}\right) \hat{s} \theta$, and $\mathbf{B} \cdot \nabla \psi \times \nabla z \simeq B_{0}^{2} / q_{0}$. Thus using Eq. (4),

$$
\begin{aligned}
\mathbf{v}_{d} \cdot \nabla A & =-\frac{v_{\|}^{2}+v_{\perp}^{2} / 2}{\Omega_{0} R_{0}}\left[\frac{\partial A}{\partial y} \cos \theta+\frac{\partial A}{\partial y} \hat{s} \theta \sin \theta+\frac{\partial A}{\partial x} \sin \theta\right] \\
& =-i k_{y} A \frac{v_{\|}^{2}+v_{\perp}^{2} / 2}{\Omega_{0} R_{0}}\left[\cos \theta+\hat{s}\left(\theta-\theta_{0}\right) \sin \theta\right]
\end{aligned}
$$


for $k_{y} \neq 0$ and $\mathbf{v}_{d} \cdot \nabla A=\left(-i k_{x} A / \Omega_{0} R_{0}\right)\left(v_{\|}^{2}+v_{\perp}^{2} / 2\right) \sin \theta$, for $k_{y}=0$. Other terms in the equations, such as $\nabla \cdot \mathrm{v}_{E}$ and $(\hat{\mathrm{b}} \cdot \nabla \hat{\mathrm{b}}) \cdot \mathrm{v}_{E}$ can also be written in the form Eq. (44).

\section{Simulation results}

We have implemented this coordinate system in nonlinear gyrofluid simulations of toroidal ITG turbulence. The simulation results are presented here to describe practical computational issues and to test some of our assumptions. It is not meant to be a complete description of our gyrofluid equations or our nonlinear results, which will be discussed more fully in later publications. Therefore, we have relegated the actual equations to the Appendix.

There are two ways to implement the boundary condition Eq. (22). Because our equations involve $\left|k_{\|}\right|$Landau damping terms (equivalent to a non-local integral operator in real space ${ }^{26}$ ), it is easiest to Fourier transform from $\theta$ to $k_{\|}$to evaluate the parallel terms. However, over the $\theta$ domain, each mode is not periodic with itself, but with a different mode. This mode will in turn be connected to another mode, etc. The most accurate way to implement the boundary condition is then to line up all the $j$ modes connected by the boundary condition onto an extended $\theta$ domain and Fourier transform in $k_{\|}$over this domain. Because the computational grid is rectangular in $k_{x}$ and $k_{y}$, the length in $\theta$ of this extended domain will be longer at lower $k_{y}$. Then we evaluate the $k_{\|}$ terms, transform back to $\theta$, and extract each $j$ mode from its position on the extended $\theta$ domain. An alternative method is to add equal length extensions in $\theta$ to each $\left(k_{x}, k_{y}\right)$ mode, as shown in Fig. 3, and copy the part of $\hat{A}_{j+\delta j, k}(\theta)$ within $-\pi N<\theta<\pi N$ onto the extension $(|\theta|>\pi N)$ of $\hat{A}_{j, k}$ before transforming to $k_{\|}$. Since we have a finite number of $k_{x}$ 's, not all modes will have a mode to connect to at $j+\delta j$. In this case $\hat{A}_{j, k}$ is zeroed in the extension, preserving periodicity. We have arranged the box so the mode amplitudes are small where this is necessary (at large $k_{y}$ ). The second approach ("the equal-length extension method") is easier to implement and to parallelize on computers, since all the FFTs in $\theta$ have the same length. But it may be linearly less accurate than the first method ("the multiply-connected method") if there are low $k_{y}$ modes which extend much further along the field line than even the extension region. (This is related to the fact that the minimum non-zero $\left|k_{\|}\right|$which can resolved for the Landau-damping operator is given by $2 \pi / L_{\|}$, 
where $L_{\|}$is the parallel box length including the extension region.) This difference is probably less important in nonlinear runs where the relevant parameter for determining the parallel box length is the parallel correlation length and not a linear mode width. In practice, we have observed no significant differences between these two methods in the nonlinear simulations done to date. The issues of an extension region (or the filtering described next) are ignorable for a particle or Vlasov simulation, since they do not require evaluation of $k_{\|}$and can directly use the boundary conditions in Sec. TV.

There is another implementation detail involving the parallel FFTs. Note that the $\theta_{0}=2 \pi N$ mode in Fig. 3 has a large amplitude at the right-hand side of the extended domain, and is not naturally periodic with itself at the left-hand end of the figure where it is zero. Fourier transforms assume periodicity, so there is effectively a sharp discontinuity for this mode across the endpoints in $\theta$ which introduces high- $k_{\|}$components into the solution. These high $k_{\|}$components are Landaudamped, but a small amount of high $k_{\|}$oscillations can propagate from the ends of the extended domain into the physical region $-N \pi<\theta<N \pi$. This high $k_{\|}$noise is reduced as the extension region is made longer, but convergence can be greatly accelerated by smoothly filtering the modes to zero near end points of the extended box. We use a filtering window which is 1 in most of the domain, and goes to zero smoothly near ends of the full (extended) domain as $2 x^{2} /\left(1+x^{4}\right)$, where $x=\left(\theta-\theta_{\text {end }}\right) / \theta_{\text {width }}$ is a normalized distance from the end points. A filtering width $\theta_{\text {width }}$ of $1 / 2$ to $1 / 4$ of the width of the extension regions appears sufficient. Thus a typical run with a physical $\theta$ domain from $-2 \pi$ to $2 \pi$ might use a fully extended domain of $-3 \pi<\theta<3 \pi$, and the filter begins to turn on within $\pi / 2$ of the endpoints at $\theta_{\text {end }}= \pm 3 \pi$. In practice, though the filtering is useful for reducing the small amount of high $k_{\|}$errors sometimes seen linearly (particularly for low $k_{y}$ modes at low shear which are extended along the field line), no statistically significant differences have been observed in the nonlinear runs with or without this filtering.

To test the small-scale assumption, we present two simulations, one with perpendicular dimensions $\left(L_{x}=85 \rho_{i}, L_{y}=100 \rho_{i}\right)$, and one with double the box size $\left(L_{x}=170 \rho_{i}, L_{y}=200 \rho_{i}\right)$. That these simulations give similar results indicates that the small flux tube may be capturing the essence of the turbulence. It is a necessary but not sufficient test, as discussed in section VIII. The 
physical parameters are taken from TFTR L-mode shot \#41309: $\eta_{i}=4, L_{n} / R=0.4, \hat{s}=1.5$, $q=2.4, T_{i}=T_{e}, \rho_{i}=.14 \mathrm{~cm}, L_{n}=103 \mathrm{~cm}$, and the computational box is centered at $r_{0}=53 \mathrm{~cm}$. The box sizes then correspond to $n_{0}=10$ for the small box and $n_{0}=5$ for the large box. Both simulations use 64 grid points along the field line coordinate $\theta$. Using 128 grid points along $\theta$ gives essentially the same results. For these runs, $N=2$, so the physical $\theta$ domain extends from $-2 \pi$ to $2 \pi$. The equal length $(\pi)$ extension method (for a total extended $\theta$ domain from $-3 \pi$ to $3 \pi$ ) was used to implement the parallel boundary condition.

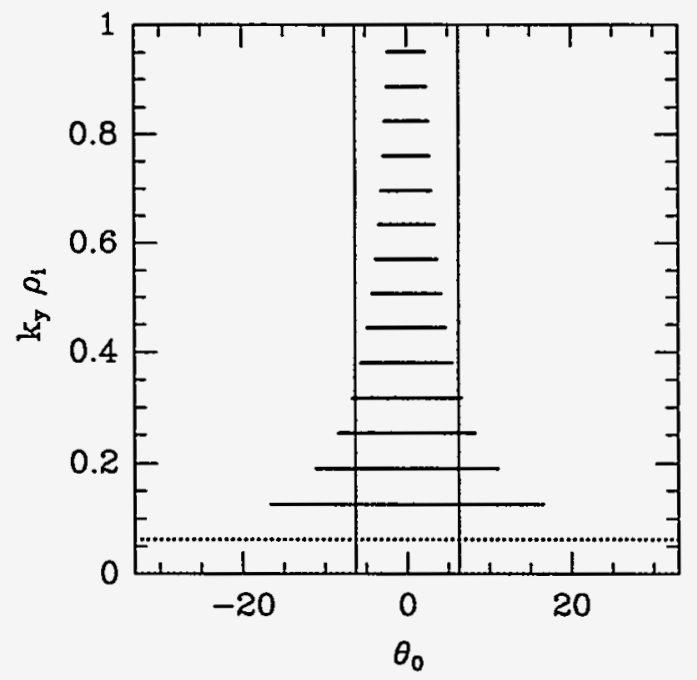

Figure 8: Distribution of $\theta_{0}$ 's for the small run. The solid lines denote the ends of the computational domain in $\theta$.

We use a spectral representation in $x$ and $y$, with $\pm 42 k_{x}$ modes and $\pm 15 k_{y}$ modes for the small simulation and $\pm 63 k_{x}$ modes and $\pm 21 k_{y}$ modes for the large simulation, not counting additional modes added at high $k$ for dealiasing. The modes are evenly spaced such that $k_{y}^{\max } \rho_{i} \approx 1$ and $k_{x}^{\min } \approx k_{y}^{\min }$, making the computational domain roughly square in $x$ and $y$. For $N>1$, it is necessary to include more $k_{x}$ 's to include unstable modes localized near $\theta= \pm 2 \pi, \pm 4 \pi$, etc., in the bad curvature regions (i.e. modes with $\theta_{0}$ 's near $\pm 2 \pi, \pm 4 \pi$, etc.). The modes tend to be localized along the field line near $\theta_{0}$, so ideally one would like to include enough $k_{x}$ 's to cover the range $-\pi N<\theta_{0}<\pi N$ for all $k_{y}$ 's. This is very expensive at high $k_{y}$, where the spacing in $\theta_{0}$ gets small, 
since $\theta_{0}=-k_{x} / \hat{s} k_{y}$. We arrange our modes in $k$ space so that the $\theta_{0}$ 's cover the $\theta$ domain for low $k_{y}$ 's, but not high $k_{y}$ 's, as shown in Fig. 8 for the smaller simulation. This implies $k_{x}^{\max } \gg k_{y}^{\max }$ for $N>1$ and $\hat{s} \approx 1$. Since most of the energy is at $k_{y} \rho_{i}<1 / 2$, the missing $\theta_{0}$ 's at high $k_{y}$ have very little effect.

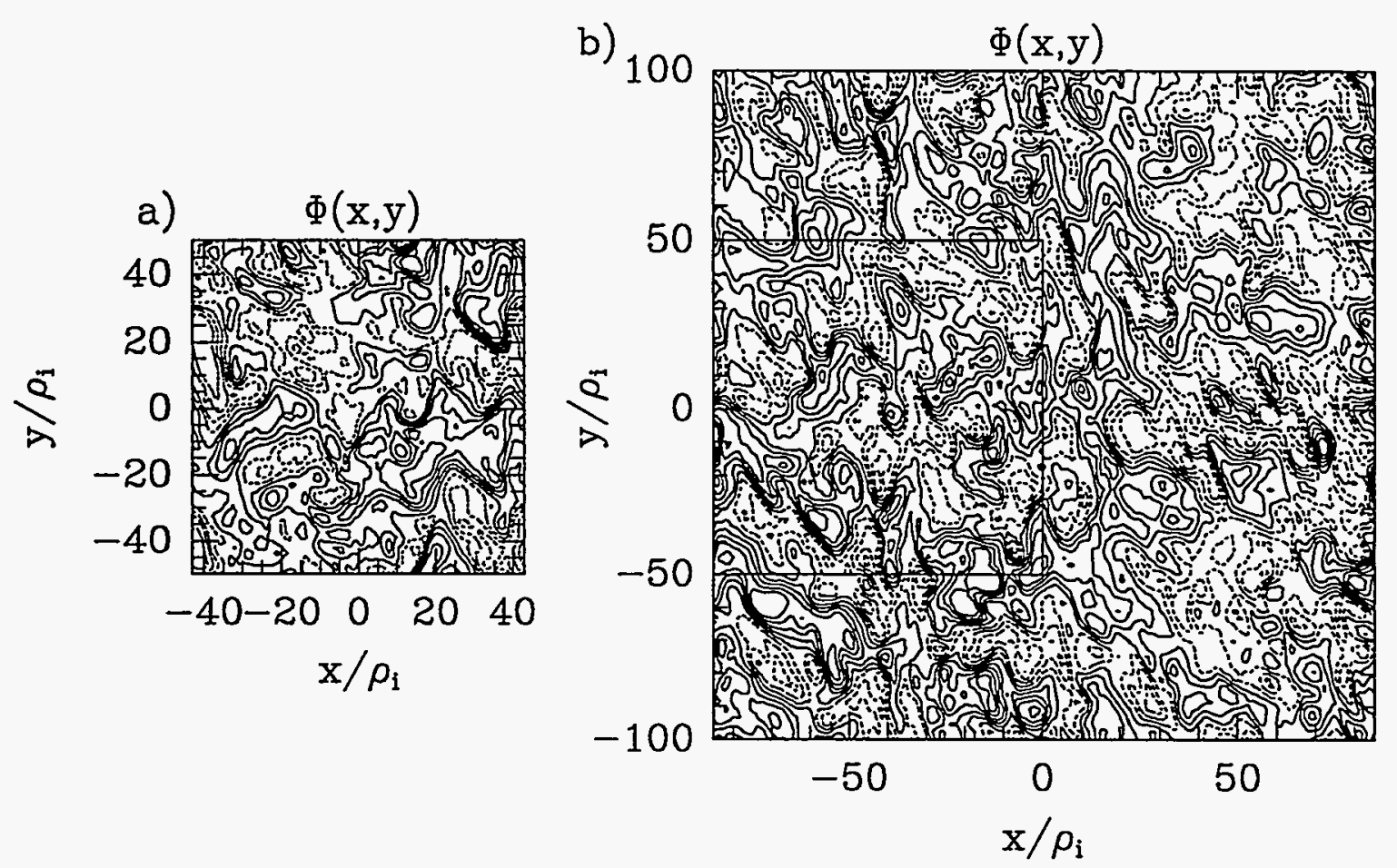

Figure 9: Contours of potential for a) small run, and b) large run. Doubling the perpendicular simulation domain did not change the dominant scale of the fluctuations.

Figure 9 shows contours of electrostatic potential in the $(x, y)$ plane at $\theta=0$ (the outer midplane of the torus), for both runs at saturation. It is apparent that although the box was doubled, the dominant scale didn't change. This is also evident from the spectra in Fig. 10, also at $\theta=0$, where $|\Phi|^{2}\left(k_{x}\right)=\sum_{k_{y}} \Phi_{k_{x}, k_{y}} \Phi_{k_{x}, k_{y}}^{*},|\Phi|^{2}\left(k_{y}\right)=\sum_{k_{x}} \Phi_{k_{x}, k_{y}} \Phi_{k_{x}, k_{y}}^{*}$, and the low resolution spectra are reduced by a factor of two to account for mode density. Although the resolution has increased, the shape and the location of the peak in the spectrum is roughly the same. These spectra are similar to BES measurements on TFTR. ${ }^{1}$ The large $k_{y}=0$ component is evidence of poloidal 

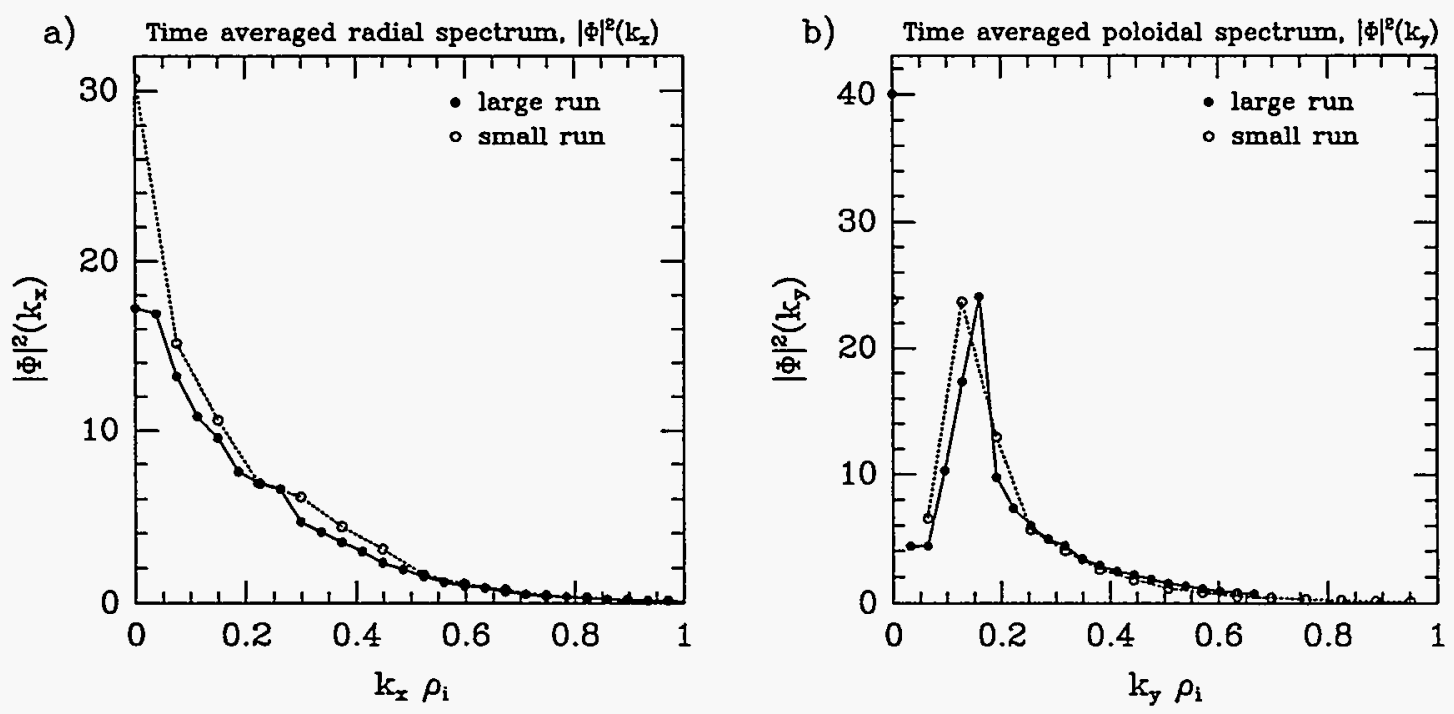

Figure 10: Potential spectra for both runs.

$\mathbf{E} \times \mathbf{B}$ rotation, as discussed in Ref. 7. Though there are some small differences in the spectra, the two runs agree within statistical fluctuations on global quantities such as the volume averaged RMS fluctuation levels and transport levels: $e \Phi / T_{i}=15 \rho_{i} / L_{n} \simeq 0.020$ and $\chi_{i}=7.4 \rho_{i}^{2} v_{t i} / L_{n}$, averaged from $t v_{t i} / L_{n}=150-300$. The evolution of $\chi_{i}$ for the two runs is shown in Fig. 11, where the statistical fluctuations are approximately $10 \%$. This level of ion heat transport is near the experimentally measured $\chi_{i}=8.8 \rho_{i}^{2} v_{t i} / L_{n}$, but these simulations ignore impurities and beams (usually a stabilizing effect), trapped electrons (destabilizing), and use our four moment model which gives lower transport than our more accurate six moment model. Nevertheless, this level of agreement is encouraging, and suggests that toroidal ITG turbulence is responsible for anomalous ion heat transport in tokamaks. The transport from these toroidal simulations is about a factor of 25 larger than sheared slab simulations for the same parameters, demonstrating the importance of toroidicity. Our toroidal simulations can be run in the sheared slab limit by taking $L_{n} / R \rightarrow 0$ and $q / \hat{s} \rightarrow 0$ so that $L_{n} / L_{s}=L_{n} \hat{s} / q R$ remains finite. We should point out that our preliminary results, Fig. $4 a$ of Ref. 7 , were high by a factor of $16 / 3$ due to a numerical error in calculating $\chi_{i}$. The remaining change is due to increased resolution. 


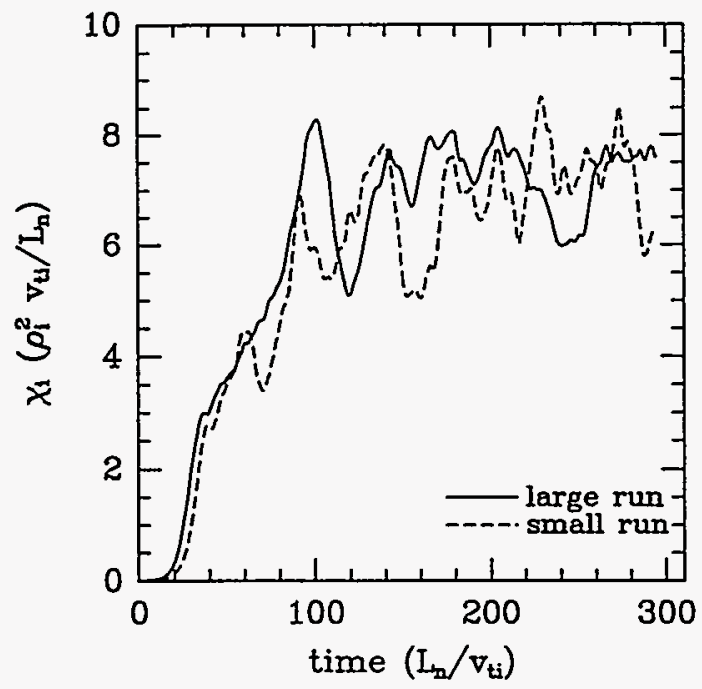

Figure 11: Evolution of $\chi_{i}$ for the large and small runs.

We have also performed tests varying the box length in the parallel direction. For these tests we have used the fully connected method to implement the parallel boundary conditions, for greatest accuracy, as described earlier in this section. Fig. 12a shows the time evolution of the volume averaged $\chi_{i}$ for two runs with box length $N=1$ and 2, i.e. $\Delta \theta=2 \pi$ and $4 \pi$, with $n_{0}=10$, and other parameters as above. Fig. 12b shows the correlation function along the field line,

$$
C(\theta, 0)=\frac{\langle\Phi(x, y, \theta) \Phi(x, y, \theta=0)\rangle}{\left\langle\Phi(x, y, \theta=0)^{2}\right\rangle}
$$

for the two runs. The averaging \langle\rangle is over $x, y$, and time once the simulation has reached a quasisteady state. If this correlation function were not averaged in $x$ and $y$ (only taken along the field line passing through $x=y=0$ ), it would return to one at $\theta= \pm 2 \pi$ for the $N=1$ run, because of periodicity. The Fourier transform of $C(\theta, 0)$ is the $k_{\|}$spectrum. As discussed in section III, since $n_{0}>1$, using a box with $-\pi<\theta<\pi,(N=1)$, can artificially constrain the parallel correlation length. There are significant correlations at $\theta \pm \pi$ for these parameters, indicating that this is the case, and that the box should be extended. These additional correlations in the $2 \pi$ box are in some way constraining the nonlinear dynamics and reducing the flux.

It is easier to test the scaling with box length at low shear, since the turbulence at $\pm 2 \pi, \pm 4 \pi$, 

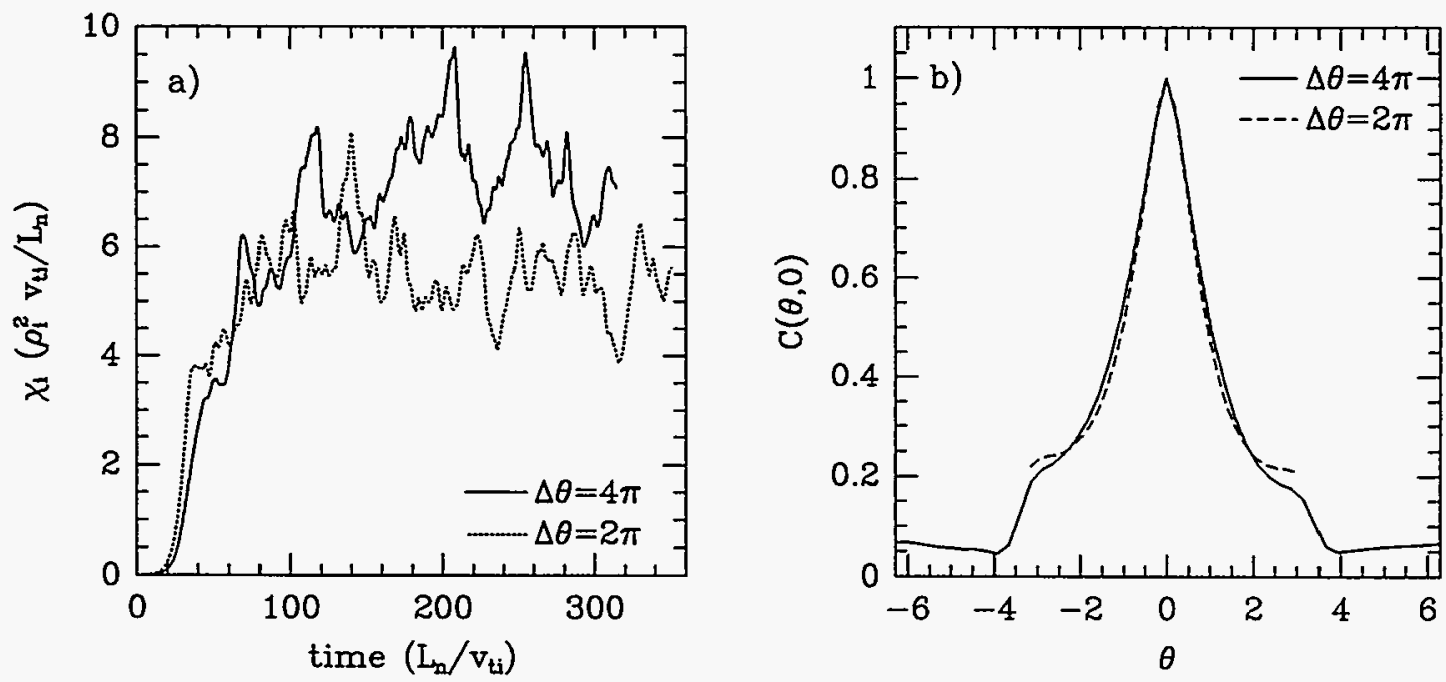

Figure 12: a) Evolution of $\chi_{i}$ for two runs with varying box length and $\hat{s}=1.5, q=2.4 \mathrm{~b}$ ) Correlation functions along the field line for the same two runs.

etc., is not at such high $k_{x}$, since $k_{x}=-k_{y} \hat{s} \theta_{0}$. This allows us to increase the box length and resolve the turbulence all along the box with fewer $k_{x}$ modes than at high shear. Also, at low shear the linear mode structure is broader in $\theta$, leading to slightly broader parallel correlation functions. Figure 13a shows the time evolution of $\chi_{i}$ in four runs with box lengths $N=1,2,3,4$ or $\Delta \theta=2 \pi, 4 \pi, 6 \pi, 8 \pi$. The physical parameters are the same as above, except $\hat{s}=0.1$ and $q=1.2$, and the perpendicular box size is $L_{x}=160 \rho_{i}, L_{y}=100 \rho_{i}$. Again, the $\Delta \theta=2 \pi$ box gives slightly lower flux, while the larger boxes all give the same flux, so the minimum box length is $\Delta \theta=4 \pi$. The correlation functions of electron density for these runs are shown in Fig. 13b, and are noticeably broader than in the higher shear cases. Using $n_{e}$ in the correlation functions removes the $k_{\|}=0$ component present in the $\Phi$ correlation functions in Fig. 12b, since $n_{e}=\Phi-\langle\Phi\rangle$ (see Appendix). For these low shear runs, the poloidal spectrum peaks at $k_{y} \rho_{i}=0.35$, so the perpendicular correlation length is smaller than in the high shear cases. This may contribute to the slightly smaller change in flux in going from $\Delta \theta=2 \pi$ to $\Delta \theta=4 \pi$, even though the parallel correlation functions are broader. The low shear runs in Fig. 13 are better resolved and are easier to run longer than the high shear runs, so we expect that a $30 \%$ change in flux is typical for ITG turbulence, where $\theta_{c} \sim 2 \pi$, when 
the artificial correlations are removed by using a longer box. We have also run with $\hat{s}=0.1$ and $q=2.4$, where $\chi_{i}=7.5 \rho_{i}^{2} v_{t i} / L_{n}$ for $\Delta \theta=4 \pi$ and $\chi_{i}=6.5 \rho_{i}^{2} v_{t i} / L_{n}$ for $\Delta \theta=2 \pi$. For $\hat{s}=0.25$ and $q=1.2$, both $\Delta \theta=2 \pi$ and $\Delta \theta=4 \pi$ give $\chi_{i}=5 \rho_{i}^{2} v_{t i} / L_{n}$, any change is within the statistical fluctuations.
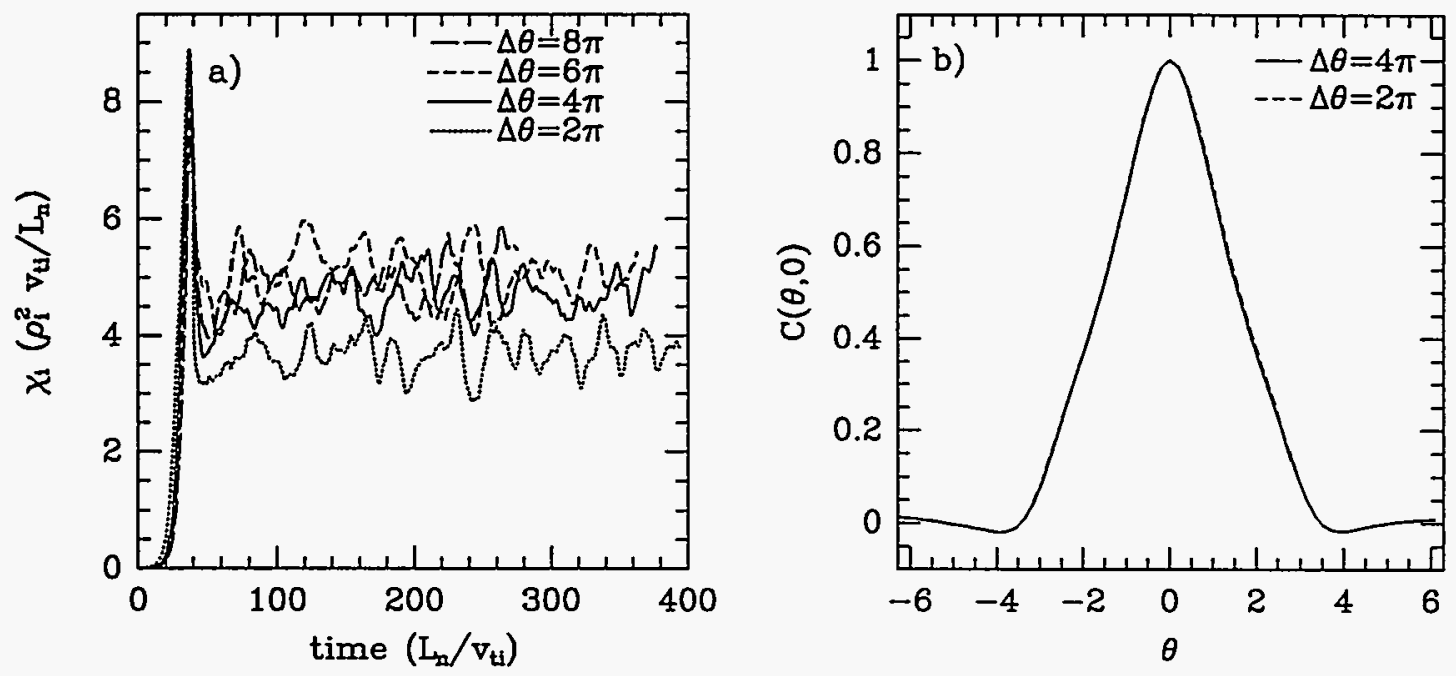

Figure 13: a) Evolution of $\chi_{i}$ for four runs with varying box length and $\hat{s}=0.1, q=1.2$. b) Correlation functions along the field line for $\Delta \theta=2 \pi$ and $4 \pi$.

\section{Discussion and Conclusions}

To summarize, we are simulating a rectangular domain in $(x, y, z)$, and using the transformation Eq. (12), this domain becomes a long, thin, twisting flux tube in a torus. The differential operators take the particularly useful forms Eq. (37-44) in the traditional tokamak model of concentric circular flux surfaces. Our flux tube approach is also applicable to general magnetic geometry, using Eqs. (2,5-6) for the differential operators. (In this case the metric coefficients $\nabla \alpha, \nabla \psi$, and $\nabla z$ need to be specified.) The boundary condition Eq. (22) can make the perturbations periodic in $\theta$, if $N=1$, which makes this representation equivalent to the ballooning representation for a coarse grid in $n$, with spacing $n_{0}$. However, when $n_{0}>1$, the box must be extended in $\theta$ to avoid non-physical correlations if the parallel correlation length is longer than $2 \pi q R$, i.e. $\theta_{c}>2 \pi$. The 
fundamental assumptions are that the correlation lengths (both parallel and perpendicular) are smaller than the box size, that the equilibrium gradients vary slowly across the small perpendicular extent of the box, and that the turbulence is local, i.e. driven only by the equilibrium gradients within the box.

The assumptions implicit in simulating a thin flux-tube subdomain should always be checked a posteriori by verifying that the simulation box is indeed at least a few correlation lengths long in each direction, so that the box is large enough for the type of turbulence under consideration. One should also verify that the results are independent of the size of the simulated flux tube (and independent of the particular choice of boundary conditions), as the flux tube is made larger than the correlation lengths. This paper has demonstrated that both conditions are met, at least for the particular cases considered in section VII. Thus our results show the existence of a gyro-Bohm scaling regime, at least for sufficiently small $\rho_{*}=\rho_{i} / L_{n}$. (Our gyrofluid equations have been scaled to the gyroradius $\rho_{i}$, and the limit $\rho_{i} / L_{n} \rightarrow 0$ taken, using the usual small-scale turbulence ordering assumptions.)

While the turbulent heat conduction from our simulations is of the right order-of-magnitude to explain experimental results from the main core region of many tokamak experiments, they have have a gyro-Bohm scaling while the actual experiments have a Bohm-scaling. ${ }^{27,28}$ (The experiments have a Bohm-like scaling with magnetic field, though the magnitude of the experimental $\chi_{i}$ is about two orders of magnitude smaller than Bohm's original formula $D=(1 / 16) c T / e B$.) Several possibilities for this discrepancy exist. One is that the experimental $\rho_{*}$, while small $\left(\sim 10^{-3}-10^{-2}\right)$, may be large enough that the radial variation of equilibrium gradients, i.e. $\omega_{*}(\psi), \eta_{i}(\psi)$, etc., or equilibrium flows, may be affecting the turbulence. For very small $\rho_{*}$ there is a scale separation between the turbulence, with scales of order $\rho_{i}$, and the equilibrium, with scale $L_{n}$, but if $\rho_{*}$ is not small enough, the turbulence may begin to feel radial variations in the equilibrium. It is interesting to note that the BES measured ${ }^{1}$ correlation length $\lambda_{c} \sim 2 \mathrm{~cm}$ is of order the geometric mean between $\rho_{i} \sim 0.15 \mathrm{~cm}$ and the minor radius $a \sim 90 \mathrm{~cm}$. Another possible explanation is that the instabilities driving the turbulence may be near marginal stability, which can mask gyro-Bohm scaling trends and, in some limits, tie the core transport scaling to edge parameters. ${ }^{29-31}$ The experiments have 
gone to great pains to keep other parameters and profiles as fixed as possible while studying the $\rho_{*}$ scaling, but a very sensitive dependence on some parameters (some of which are hard to measure) could also mask, at least partially, a gyro-Bohm scaling. Another possible explanation might involve non-local turbulence, where fluctuations radially propagate a significant distance from where they were generated by an instability, an effect which is currently under debate. ${ }^{32,33}$

Numerical studies of some of these effects do not necessarily require simulating the whole tokamak. Rather, one could consider a somewhat thicker flux-tube than usual, and include the radial variations of $\omega_{*}(\psi), \eta_{i}(\psi)$, and other plasma parameters over the simulated region in the governing gyrofluid (or gyrokinetic) equations. Even if simulating the full torus radially, field-line coordinates are useful to allow a coarser grid in the parallel direction, and a coarser grid in the toroidal mode number $n$. When the equilibrium profiles are assumed to be constant, so $L_{n}, L_{T}$, etc. do not vary radially (as assumed in our simulations), the linear eigenmodes are unbounded radially. In ballooning terminology, the solutions of the zeroth order eigenmode equation in $1 / n q$ are independent of $\psi$. In a real tokamak, however, the radial profile variation determines the radial extent of the linear modes, and this radial structure is determined from a higher order equation in $1 / n q$. Recently, there has been renewed interest in the solution for this radial envelope, and the modifications to the zeroth order eigenfrequencies. ${ }^{23}$ For longer wavelength global modes, the linear radial mode structure is also determined by the radial variation of equilibrium gradients. ${ }^{34}$ An alternative way to include these effects is to still use Eq. (7) to represent the perturbations, but to include the radial variation of equilibrium profiles. This $\psi$ dependence will linearly couple different $j$ modes in Eq. (7), which are uncoupled when the profiles have constant gradients. Then the superposition of different $j$ (i.e. $k_{x}$ ) modes will determine the radial envelope of the true linear mode. However, since the nonlinear $\mathbf{E} \times \mathbf{B}$ coupling of the various $\hat{A}_{j, k}$ modes is usually much stronger than this linear coupling, it is likely that the precise radial linear mode shape is subdominant, and that the radial scale length of the turbulence is set by nonlinear processes, as suggested by Cowley, et al., ${ }^{6}$ and Mattor. ${ }^{35}$ Comparing the order of magnitude of these effects in, for example, the density equation, we have:

$$
\frac{1}{n_{0}} \mathbf{v}_{E} \cdot \nabla n \sim \rho_{i} v_{t i} k_{\perp}^{2} \frac{e \Phi}{T_{i}} \frac{n}{n_{0}}
$$




$$
\frac{1}{n_{0}} \mathbf{v}_{E} \cdot \nabla n_{0}(x) \sim \frac{\rho_{i} v_{t i} k_{\perp}}{L_{n}} \frac{e \Phi}{T_{i}}\left[1+\mathcal{O}\left(x / L_{n}\right)+\mathcal{O}\left(x^{2} / L_{n}^{2}\right)+\ldots\right] .
$$

The nonlinear term is of the same order as the $x$ independent linear term (i.e. the $\omega_{*}\left(\psi_{0}\right)$ term) in the standard gyrokinetic ordering, where $n / n_{0} \sim \rho_{i} / L_{n}$ and $k_{\perp} \rho_{i} \sim 1$. While the linear mode widths are broad, the typical turbulent eddy size is not much larger than $\sim 10 \rho_{i}$, so it would seem that the $x$-dependent term $\left(\propto \partial \omega_{*} / \partial \psi\right)$ can usually be ignored.

Equilibrium sheared zonal flows $\left(k_{y}=0, k_{z}=0, k_{x} \neq 0\right.$ flows which cause flux surfaces to rotate) can be included in our representation in several ways (one of which is presented in Ref. 20), though we have not yet implemented them in our simulations. Such sheared flows can be important, particularly near the plasma edge where they appear to be responsible for the H-mode transition. ${ }^{36}$ Though we are presently neglecting equilibrium-scale zonal flows, we do include the higher $k_{\tau}$ components of the zonal flows which are generated by the turbulence itself.

For typical tokamak parameters, our reduced simulation volume can represent large computational savings. We compare rough scalings with some other methods; the results are only order of magnitude estimates. Perhaps the most straightforward way to simulate a tokamak is with the " $m, n, r$ " representation:

$$
\Phi(\psi, \theta, \zeta)=\sum_{m, n} e^{i n \zeta-i m \theta} \hat{\Phi}_{m, n}(\psi)
$$

Since we are interested in simulating fine-scale turbulence, we need to resolve perpendicular scales of order $\rho_{i}$. If we are simulating a full torus, the range of $m$ 's must be $m \in\left(0, \pm 1, \ldots, \pm a / \rho_{i}\right)$. To resolve the long parallel structure, the range of $n$ 's must be $n \in\left(0, \pm 1, \ldots, \pm a / q \rho_{i}\right)$, where $q$ is a representative value, around 2. The radial grid for $\hat{\Phi}_{m, n}(\psi)$ must resolve $\rho_{i}$ and span the minor radius, so $r=l \Delta_{r}$, where $\Delta_{\tau} \sim \rho_{i}$ and $l \in\left(0,1, \ldots, a / \rho_{i}\right)$. This gives the total number of grid points, for $a \sim 10^{3} \rho_{i}$,

$$
N_{m, n, r} \sim \frac{1}{q}\left(\frac{a}{\rho_{i}}\right)^{3} \sim 10^{9}
$$

This is the same as expected from a computational grid in the physical $r, \theta, \zeta$ space, where the $\zeta$ grid can be $1 / q$ coarser than the $r$ or $\theta$ directions.

By simulating a thin toroidal annulus in $r$, but still going all the way around in $\theta$ and $\zeta$, the number of radial grid points is reduced by $\Delta r / a$, which for our simulations is typically $1 / 10$. 
Further, aligning the grid points with the field lines (or nearly aligning with the field, as proposed by Dimits ${ }^{15}$ ) reduces the necessary resolution in this direction. We have found that 64 grid points along the field line is adequate, so the number of grid points for a thin annulus with a field-aligned coordinate is:

$$
N_{m, n, r} \sim 64\left(\frac{a}{\rho_{i}}\right)^{2} \frac{\Delta r}{a} \sim 10^{7} .
$$

The next level of reduction is to also exploit the small perpendicular correlation length in the poloidal direction, which brings us to our twisting flux tube:

$$
N_{\text {flux tube }} \sim 64\left(\frac{a}{\rho_{i}}\right)^{2} \frac{\Delta r}{a} \frac{\Delta y}{a} \sim 10^{6},
$$

so for the simulation in Fig. 9a, counting modes included for dealiasing, we used:

$$
N \sim 64 \times 128 \times 48 \sim 4 \times 10^{5} .
$$

Kotschenreuther and Wong ${ }^{14}$ have proposed using the representation:

$$
\Phi(r, \theta, \zeta)=\sum_{j, l} e^{i l\left(m_{0} \theta-n_{0} \zeta\right)} e^{i j \theta} \hat{\Phi}_{j, l}\left(r-r_{0}\right)
$$

which has many similarities to our representation. It is periodic in $\zeta$ with period $2 \pi / n_{0}$ and in $\theta$ over $2 \pi$, and is therefore simulating a wedge of a toroidal annulus when the $r$ domain is small. Thus Eq. (47) is numerically as efficient as the one described in this paper, however, if $\theta_{c}>2 \pi$ false correlations along the parallel direction will be introduced, as discussed in section III. It is not obvious how to remedy this problem with Eq. (47), but with our approach one simply uses a longer box, i.e. $N>1$.

The "quasiballooning" approach of Dimits ${ }^{15}$ shares similar computational advantages to our method. Indeed, the quasiballooning (almost-field-line coordinates) method has many similarities to the field-line coordinates approach of Roberts and Taylor, ${ }^{5}$ and Cowley, et al., ${ }^{6}$ upon which our paper is based, though the quasiballooning method emphasizes the perspective of a real-space radial grid while we use discrete Fourier transforms for the radial direction which illustrate its relation to the usual ballooning transformation. We have shown that physical periodicity in $\theta$ can be also be implemented with our approach, but that there are cases where one should forgo physical periodicity in favor of a longer box (i.e., $N>1$ ) to avoid false parallel correlations. As described in 
Sec. III, simulating only $1 / n_{0}$ of the toroidal direction is often justified by the short perpendicular correlation lengths of the turbulence, but that makes a perturbation extended along a field-line $n_{0}$ times as likely to "bite it's tail", which should be compensated for by making the box longer than a parallel correlation length. In principle, $N=1$ simulations should eventually converge as the box is made large enough in the perpendicular directions (so that $n_{0} \rightarrow 1$ ), but from the runs we have done it appears that faster convergence is obtained by allowing the box to be longer than a parallel correlation length as well, thus consistently following the principle that the simulation domain should be longer than the correlation lengths in all three directions.

\section{Acknowledgements}

The authors thank R. E. Waltz and G. D. Kerbel for useful discussions. Our toroidal nonlinear gyrofluid code grew out of the earlier slab gyrofluid code developed by W. Dorland, ${ }^{9,16}$ who provided helpful computational advice and physics discussions. M.A.B. also thanks Q. P. Liu, D. P. Coster, and M. Artun for further useful discussions and computational advice. M.A.B. and G.W.H. thank the TFTR project for supporting this work, and the National Energy Research Supercomputer Center for computing resources. This work supported in part by the High Performance Computing and Communications Initiative (HPCCI) Grand Challenge Numerical Tokamak Project, by USDoE contract No. DE-AC02-76-CH0-3073, contract No. DE-FG03-93ER54224, and by a National Science Foundation Graduate Fellowship.

\section{Appendix. Toroidal gyrofluid equations}

The equations used in the simulations are briefly summarized here, they are discussed in more detail in Refs. 7, 37-40. In these simulations, we evolve four moments of the gyrokinetic equation, the perturbed guiding center density, parallel flow, parallel temperature, and perpendicular temperature, with closure approximations to model the effects of parallel resonances, toroidal res-

onances, and FLR. Here we ignore collisions and particle trapping (i.e. $\hat{\mathrm{b}} \cdot \nabla B=0$ ), although we have developed models of these effects, and have extended this model to up to six moments. ${ }^{7}$ Using 
the normalizations in Ref. 37, the dynamical equations are:

$$
\begin{aligned}
& \frac{d n}{d t}+\hat{\mathbf{b}} \cdot \nabla u_{\|}+\left(1+\frac{\eta_{i}}{2} \hat{\nabla}_{\perp}^{2}\right) \frac{\partial \Psi}{\partial y}+\left(\frac{1}{2} \hat{\nabla}_{\perp}^{2} \mathbf{v}_{\Psi}\right) \cdot \nabla T_{\perp}=-i \omega_{d}\left(T_{\|}+T_{\perp}+2 n+2 \Psi\right) \\
& \frac{d u_{\|}}{d t}+\hat{\mathbf{b}} \cdot \nabla\left(T_{\|}+n+\Psi\right)=-2 i \omega_{d} \nu_{5 i} u_{\|}-2\left|\omega_{d}\right| \nu_{5 r} u_{\|}, \\
& \frac{d T_{\|}}{d t}+2 \hat{\mathbf{b}} \cdot \nabla u_{\|}+\sqrt{2}\left|k_{\|}\right| \chi_{\|} T_{\|}+\eta_{i} \frac{\partial \Psi}{\partial y}= \\
& \quad-2 i \omega_{d}\left[\left(3+\nu_{1 i}\right) T_{\|}+\nu_{2 i} T_{\perp}+n+\Psi\right]-2 \nu_{1 r}\left|\omega_{d}\right| T_{\|}-2 \nu_{2 r}\left|\omega_{d}\right| T_{\perp}, \\
& \frac{d T_{\perp}}{d t}+\sqrt{2}\left|k_{\|}\right| \chi_{\perp}\left(T_{\perp}+\frac{1}{2} \hat{\nabla}_{\perp}^{2} \Psi\right)+\left[\frac{1}{2} \hat{\nabla}_{\perp}^{2}+\eta_{i}\left(1+\hat{\hat{\nabla}}_{\perp}^{2}\right)\right] \frac{\partial \Psi}{\partial y}+\left(\frac{1}{2} \hat{\nabla}_{\perp}^{2} \mathbf{v}_{\Psi}\right) \cdot \nabla n+\left(\hat{\hat{\nabla}}_{\perp}^{2} \mathbf{v}_{\Psi}\right) \cdot \nabla T_{\perp} \\
& \quad=-2 i \omega_{d}\left[\nu_{3 i} T_{\|}+\left(2+\nu_{4 i}\right) T_{\perp}+\frac{n}{2}+\frac{1}{2}\left(1+\frac{3}{2} \hat{\nabla}_{\perp}^{2}\right) \Psi\right]-2 \nu_{3 r}\left|\omega_{d}\right| T_{\|}-2 \nu_{4 r}\left|\omega_{d}\right| T_{\perp} .
\end{aligned}
$$

The total time derivative includes the $\mathbf{E} \times \mathbf{B}$ nonlinearities, $d / d t=\partial / \partial t+\mathbf{v}_{\Psi} \cdot \nabla$. The gyroaveraged potential and $\mathbf{E} \times \mathbf{B}$ drift are $\Psi=\Gamma_{0}^{1 / 2} \Phi$ and $\mathbf{v}_{\Psi}=\hat{\mathbf{b}} \times \nabla \Psi$, respectively. The toroidal drift terms have been written using $i \omega_{d} \equiv\left(v_{t}^{2} / \Omega B^{2}\right) \mathrm{B} \times \nabla B \cdot \nabla$.

The closure coefficients for this set of moments are chosen to provide an accurate approximation to the linear kinetic response. The parallel closure coefficients are $\chi_{\|}=2 / \sqrt{\pi}$ and $\chi_{\perp}=1 / \sqrt{\pi}$. The toroidal closure coefficients have both dissipative and reactive pieces, and written in the form $\nu=\left(\nu_{r}, \nu_{i}\right)=\nu_{r}+i \nu_{i}\left|\omega_{d}\right| / \omega_{d}$, they are $\nu_{1}=(1.93,-.39), \nu_{2}=(.24,1.29), \nu_{3}=(-1.40, .47)$, $\nu_{4}=(-.14,-1.75)$, and $\nu_{5}=(.76,-.98)$.

We assume adiabatic electron response, $n_{e}=\tau(\Phi-\langle\Phi\rangle)$, where $\langle\Phi\rangle(\psi)=\left(\int d \alpha d z J|\nabla \psi| \Phi\right) /$ $\left(\int d \alpha d z J|\nabla \psi|\right)$ is a flux surface average. In circular concentric geometry, this becomes $\langle\Phi\rangle=$ $\left(4 \Delta y z_{0}\right)^{-1} \int d y d z\left(R / R_{0}\right) \Phi(x, y, z)$, and is only nonzero for the $k_{y}=0$ components. This form of the adiabatic electron response prevents radial electron flow which would short out the electric field responsible for the nonlinearly generated sheared poloidal flows which are essential for saturation. ${ }^{7}$ The gyrokinetic quasineutrality constraint is, with $\tau=T_{i} / T_{e}$ :

$$
n_{e}=\bar{n}_{i}+\left(\Gamma_{0}-1\right) \Phi
$$

where $\bar{n}_{i}$ is the ion density, which is related to the ion guiding center density and perpendicular temperature by the FLR closure relation in Ref. 37, yielding:

$$
\tau(\Phi-\langle\Phi\rangle)=\frac{\Gamma_{0}^{1 / 2}}{D(b)}\left[N(b) n+\frac{1}{2} \hat{\nabla}_{\perp}^{2} T_{\perp}\right]+\left(\Gamma_{0}-1\right) \Phi .
$$


The functions $N(b), D(b)$, where $b=k_{\perp}^{2} \rho_{i}^{2}$, and the operators $\hat{\nabla}_{\perp}$ and $\hat{\vec{\nabla}}_{\perp}$ model FLR effects, and explicit forms are given in Ref. 37. Since this equation involves both $\Phi$ and $\langle\Phi\rangle$, we use the following procedure to determine $\Phi$, given $n$ and $T_{\perp}$. In general, the coefficients in this equation can be functions of the field line coordinate, so writing $\Phi=\langle\Phi\rangle+\delta \Phi$, and solving for $\delta \Phi$ gives:

$$
\delta \Phi=\frac{\bar{n}_{i}+\left(\Gamma_{0}-1\right)\langle\Phi\rangle}{\tau+1-\Gamma_{0}}
$$

Averaging both sides, since $\langle\delta \Phi\rangle=0$, and solving for $\langle\Phi\rangle$ gives:

$$
\langle\Phi\rangle=\left\langle\frac{\bar{n}_{i}}{\tau+1-\Gamma_{0}}\right\rangle /\left\langle\frac{\left(1-\Gamma_{0}\right)}{\tau+1-\Gamma_{0}}\right\rangle .
$$

Now that $\langle\Phi\rangle$ is determined, we use this expression in the quasineutrality constraint to obtain $\Phi$. 


\section{References}

${ }^{1}$ R. J. Fonck, G. Cosby, R. D. Durst, S. F. Paul, N. Bretz, S. Scott, E. Synakowski, and G. Taylor, Phys. Rev. Lett. 70, 3736 (1993).

${ }^{2}$ E. Mazzucato and R. Nazikian, Phys. Rev. Lett. 71, 1840 (1993).

${ }^{3}$ S. Zweben and S. S. Medley, Phys. Fluids B 1, 2058 (1989).

${ }^{4}$ G. W. Hammett, M. A. Beer, J. C. Cummings, W. Dorland, W. W. Lee, H. E. Mynick, S. E. Parker, R. A. Santoro, M. Artun, H. P. Furth, T. S. Hahm, G. Rewoldt, W. M. Tang, R. E. Waltz, G. D. Kerbel, and J. Milovich, in Plasma Physics and Controlled Nuclear Fusion Research, 1994, paper IAEA-CN-60/D-2-II-1.

${ }^{5}$ K. V. Roberts and J. B. Taylor, Phys. Fluids 8, 315 (1965).

${ }^{6}$ S. C. Cowley, R. M. Kulsrud, and R. Sudan, Phys. Fluids B 3, 2767 (1991).

${ }^{7}$ G. W. Hammett, M. A. Beer, W. Dorland, S. C. Cowley, and S. A. Smith, Plasma Phys. Controlled Fusion 35, 973 (1993).

${ }^{8}$ M. D. Kruskal and R. M. Kulsrud, Phys. Fluids 1, 265 (1958).

${ }^{9}$ W. Dorland, G. W. Hammett, T. S. Hahm, and M. A. Beer, in U. S.-Japan Workshop on Ion Temperature Gradient Driven Turbulent Transport, edited by W. Horton, M. Wakatani, and A. Wootton, (AIP, New York, 1993), p. 344.

${ }^{10}$ B. Cohen, T. J. Williams, A. M. Dimits, and J. A. Byers, Phys. Fluids B 5, 2967 (1993).

${ }^{11}$ A. Hasegawa and M. Wakatani, Phys. Rev. Lett. 59, 1581 (1987).

${ }^{12}$ B. A. Carreras, V. E. Lynch, and L. Garcia, Phys. Fluids B 3, 1438 (1991).

${ }^{13}$ P. H. Diamond and Y. B. Kim, Phys. Fluids B 3, 1626 (1991).

${ }^{14}$ M. Kotschenreuther and H. V. Wong, private communication (1991). 
${ }^{15}$ A. M. Dimits, Phys. Rev. E 48, 4070 (1993).

${ }^{16}$ W. Dorland, Ph.D. Thesis, Princeton University (1993).

${ }^{17}$ R. B. White, Theory of Tokamak Plasmas (North Holland, Amsterdam, 1989).

${ }^{18}$ J. M. Greene and J. L. Johnson, Phys. Fluids 5, 510 (1962).

${ }^{19}$ R. E. Waltz and A. H. Boozer, Phys. Fluids B 5, 2201 (1993).

${ }^{20}$ R. E. Waltz, G. D. Kerbel, and J. Milovich, Phys. Plasmas 1, 2229 (1994).

${ }^{21}$ M. C. Zarnstorff, S. Batha, A. Janos, F. L. Levinton, and the TFTR Group, in Local Transport Studies in Fusion Plasmas, edited by J. D. Callen, G. Gorini, and E. Sindoni, (Societa Italiana di Fisica, Bologna, 1993), p. 257.

${ }^{22}$ J. W. Connor, R. J. Hastie, and J. B. Taylor, Proc. R. Soc. London A 365, 1 (1979); A. H. Glasser, in Proceedings of the Finite Beta Theory Workshop, Varenna, 1977, edited by B. Coppi and W. Sadowski (U. S. Department of Energy, CONF-7709167, 1977), p. 55; Y. C. Lee and J. W. Van Dam, ibid., p. 93.

${ }^{23}$ J. W. Connor, J. B. Taylor, and H. R. Wilson, Phys. Rev. Lett. 70, 1803 (1993); J. B. Taylor, J. W. Connor, and H. R. Wilson, Plasma Phys. Controlled Fusion 35, 1063 (1993); N. Mattor, Phys. Plasmas 1, 245 (1994).

${ }^{24}$ R. D. Hazeltine and W. A. Newcomb, Phys. Fluids B 2, 7 (1990).

${ }^{25}$ E. A. Frieman and Liu Chen, Phys. Fluids 25, 502 (1982).

${ }^{26}$ G. W. Hammett and F. W. Perkins, Phys. Rev. Lett. 64, 3019 (1990).

${ }^{27}$ S. D. Scott, C. W. Barnes, D. M. Mikkelsen, F. W. Perkins, M. G. Bell, R. E. Bell, C. E. Bush, D. E. Ernst, E. D. Fredrickson, B. Grek, K. W. Hill, A. C. Janos, F. C. Jobes, D. W. Johnson, D. K. Mansfield, D. K. Owens, H. Park, S. F. Paul, A. T. Ramsey, J. Schivell, B. C. Stratton, E. J. Synakowski, W. M. Tang, and M. C. Zarnstorff, in Plasma Physics and Controlled Nuclear Fusion Research, 1992 (International Atomic Energy Agency, Vienna, 1993), Vol. 3, p. 427. 
${ }^{28}$ F. W. Perkins, C. W. Barnes, D. W. Johnson, S. D. Scott, M. C. Zarnstorff, M. G. Bell, R. E. Bell, C. E. Bush, B. Grek, K. W. Hill, D. K. Mansfield, H. Park, A. T. Ramsey, J. Schivell, B. C. Stratton, and E. Synakowski, Phys. Fluids B 5, 477 (1993).

${ }^{29}$ P. W. Terry, J.-N. Leboeuf, P. H. Diamond, D. R. Thayer, J. E. Sedlak, and G. S. Lee, Phys. Fluids 31, 2920 (1988).

${ }^{30}$ H. Biglari, P. H. Diamond, and M. N. Rosenbluth, Phys. Fluids B 1, 109 (1989).

${ }^{31}$ M. Kotschenreuther, et al., in Plasma Physics and Controlled Nuclear Fusion Research, 1992 (International Atomic Energy Agency, Vienna, 1993), Vol. 2, p. 11.

${ }^{32}$ X. Garbet, L. Laurent, J. P. Roubin, A. Samain, in Plasma Physics and Controlled Nuclear Fusion Research, 1992 (International Atomic Energy Agency, Vienna, 1993), Vol. 2, p. 213.

${ }^{33}$ N. Mattor and P. H. Diamond, Phys. Rev. Lett. 72, 486 (1994).

${ }^{34}$ W. M. Tang and G. Rewoldt, Phys. Fluids B 5, 2451 (1993).

${ }^{35}$ N. Mattor, Phys. Fluids B 3, 1913 (1991).

${ }^{36}$ H. Biglari, P.H. Diamond, and P.W. Terry, Phys. Fluids B 2, 1 (1990).

${ }^{37}$ W. Dorland and G. W. Hammett, Phys. Fluids B 5, 812 (1993).

${ }^{38}$ R. E. Waltz, R. R. Dominguez, and G. W. Hammett, Phys. Fluids B 4, 3138 (1992).

${ }^{39}$ G. M. Staebler, R. E. Waltz, M. A. Beer, W. Dorland, R. R. Dominguez, C. M. Greenfield, G. W. Hammett, F. L. Hinton, G. D. Kerbel, TK. Kurki-Suonio, and J. C. Wiley, in Plasma Physics and Controlled Nuclear Fusion Research, 1992 (International Atomic Energy Agency, Vienna, 1993), Vol. 2, p. 329.

${ }^{40}$ M. A. Beer, G. W. Hammett, W. Dorland, and S. C. Cowley, Bull. Am. Phys. Soc. 37, 1478 (1992). 
Dr. F. Paoloni, Univ. of Wollongong, AUSTRALIA

Prof. R.C. Cross, Univ. of Sydney, AUSTRALIA

Plasma Research Lab., Australian Nat. Univ., AUSTRALIA

Prof. I.R. Jones, Flinders Univ, AUSTRALIA

Prof. F. Cap, Inst. for Theoretical Physics, AUSTRIA

Prof. M. Heindler, Institut für Theoretische Physik, AUSTRIA

Prof. M. Goossens, Astronomisch Instituut, BELGIUM

Ecole Royale Militaire, Lab. de Phy. Plasmas, BELGIUM

Commission-European, DG. XII-Fusion Prog., BELGIUM

Prof. R. Bouciqué, Rijksuniversiteit Gent, BELGIUM

Dr. P.H. Sakanaka, Instituto Fisica, BRAZIL

Prof. Dr. I.C. Nascimento, Instituto Fisica, Sao Paulo, BRAZIL Instituto Nacional De Pesquisas Espaciais-INPE, BRAZIL Documents Office, Atomic Energy of Canada Ltd., CANADA Ms. M. Morin, CCFM/Tokamak de Varennes, CANADA Dr. M.P. Bachynski, MPB Technologies, Inc., CANADA Dr. H.M. Skarsgard, Univ. of Saskatchewan, CANADA Prof. J. Teichmann, Univ. of Montreal, CANADA Prot. S.R. Sreenivasan, Univ. of Calgary, CANADA Prof. T.W. Johnston, INRS-Energie, CANADA Dr. R. Bolton, Centre canadien de fusion magnétique, CANADA Dr. C.R. James, Univ. of Alberta, CANADA Dr. P. Lukác, Komenského Universzita, CZECHO-SLOVAKIA The Librarian, Culham Laboratory, ENGLAND Library, R61, Rutherford Appleton Laboratory. ENGLAND Mrs. S.A. Hutchinson, JET Library, ENGLAND Dr. S.C. Sharma, Univ. of South Pacific, FIJI ISLANDS P. Mähönen, Univ. of Helsinki, FINLAND Prof. M.N. Bussac, Ecole Polytechnique,. FRANCE C. Mouttet, Lab. de Physique des Milieux lonisés, FRANCE J. Radet, CEN/CADARACHE - Bat 506, FRANCE Prof. E. Economou, Univ. of Crete, GREECE Ms. C. Rinni, Univ. of loannina, GREECE Preprint Library, Hungarian Academy of Sci., HUNGARY Dr. B. DasGupta, Saha Inst. of Nuclear Physics, INDIA Dr. P. Kaw, Inst. for Plasma Research, INDIA Dr. P. Rosenau, Israel Inst. of Technology, ISRAEL Librarian, International Center for Theo Physics, ITALY Miss C. De Palo, Associazione EURATOM-ENEA , ITALY Dr. G. Grosso, Istituto di Fisica del Plasma, ITALY Prof. G. Rostangni, Istituto Gas lonizzati Del Cnr, ITALY
Dr. H. Yamato, Toshiba Res \& Devel Center, JAPAN

Prof. I. Kawakami, Hiroshima Univ., JAPAN

Prof. K. Nishikawa, Hiroshima Univ., JAPAN

Librarian, Naka Fusion Research Establishment, JAERI, JAPAN

Director, Japan Atomic Energy Research Inst.. JAPAN

Prof. S. Itoh, Kyushu Univ., JAPAN

Research Info. Ctr., National Instit. for Fusion Science, JAPAN

Prof. S. Tanaka, Kyoto Univ., JAPAN

Library, Kyoto Univ., JAPAN

Prof. N. Inoue, Univ. of Tokyo, JAPAN

Secretary, Plasma Section, Electrotechnical Lab., JAPAN

Dr. O. Mitarai, Kumamoto Inst. of Technology, JAPAN

Dr. G.S. Lee, Korea Basic Sci. Ctr., KOREA

J. Hyeon-Sook, Korea Atomic Energy Research Inst., KOREA

D.I. Choi, The Korea Adv. Inst. of Sci. \& Tech., KOREA

Prof. B.S. Liley, Univ. of Waikato, NEW ZEALAND Inst of Physics, Chinese Acad Sci PEOPLE'S REP. OF CHINA Library, Inst. of Plasma Physics, PEOPLE'S REP. OF CHINA Tsinghua Univ. Library, PEOPLE'S REPUBLIC OF CHINA Z. Li, S.W. Inst Physics, PEOPLE'S REPUBLIC OF CHINA Prof. J.A.C. Cabral, Instituto Superior Tecnico, PORTUGAL Prof. M.A. Hellberg, Univ. of Natal, S. AFRICA Prof. D.E. Kim, Pohang Inst. of Sci. \& Tech., SO. KOREA Prof. C.I.E.M.A.T, Fusion Division Library, SPAIN Dr. L. Stenflo, Univ. of UMEA, SWEDEN Library, Royal Inst. of Technology, SWEDEN Prof. H. Wilhelmson, Chalmers Univ. of Tech., SWEDEN Centre Phys. Des Plasmas, Ecole Polytech, SWITZERLAND Bibliotheek, Inst. Voor Plasma-Fysica, THE NETHERLANDS Asst. Prof. Dr. S. Cakir, Middle East Tech. Univ., TURKEY Dr. V.A. Glukhikh,Sci. Res. Inst. Electrophys.I Apparatus, USSR Dr. D.D. Ryutov, Siberian Branch of Academy of Sci., USSR

Dr. G.A. Eliseev, I.V. Kurchatov Inst., USSR Librarian, The Ukr.SSR Academy of Sciences, USSR Dr. L.M. Kovrizhnykh, Inst. of General Physics, USSR Kemforschungsanlage GmbH, Zentralbibliothek, W. GERMANY Bibliothek, Inst. Für Plasmaforschung, W. GERMANY Prof. K. Schindler, Ruhr-Universitát Bochum, W. GERMANY Dr. F. Wagner, (ASDEX), Max-Planck-Institut, W. GERMANY Librarian, Max-Planck-Institut, W. GERMANY 